University of Nebraska - Lincoln

DigitalCommons@University of Nebraska - Lincoln

1999

\title{
Kinetic features of alloy ordering with many types of ordered domain: D03-type orderings
}

\author{
Kirill D. Belashchenko \\ 'Kurchatov Institute' Russian Research Centre, belashchenko@unl.edu \\ G D Samolyuk \\ 'Kurchatov Institute' Russian Research Centre \\ V G Vaks \\ 'Kurchatov Institute' Russian Research Centre, vaks@mbslab.kiae.ru
}

Follow this and additional works at: https://digitalcommons.unl.edu/physicsbelashchenko

Belashchenko, Kirill D.; Samolyuk, G D; and Vaks, V G, "Kinetic features of alloy ordering with many types of ordered domain: D03-type orderings" (1999). Kirill Belashchenko Publications. 4.

https://digitalcommons.unl.edu/physicsbelashchenko/4

This Article is brought to you for free and open access by the Research Papers in Physics and Astronomy at DigitalCommons@University of Nebraska - Lincoln. It has been accepted for inclusion in Kirill Belashchenko Publications by an authorized administrator of DigitalCommons@University of Nebraska - Lincoln. 


\title{
Kinetic features of alloy ordering with many types of ordered domain: $\mathrm{D0}_{3}$-type orderings
}

\author{
K D Belashchenko, G D Samolyuk and V G Vaks \\ 'Kurchatov Institute' Russian Research Centre, Moscow 123182, Russia \\ Received 9 February 1999, in final form 7 September 1999
}

\begin{abstract}
The earlier-described master equation approach to the configurational kinetics of nonequilibrium alloys is used to study kinetic features of 'multivariant' orderings for which more than two types of ordered domain are possible. To this end we make extensive simulations of various phase transformations involving $\mathrm{D}_{3}$-type orderings for a number of alloy models. The microscopic structure of various antiphase boundaries in the $\mathrm{DO}_{3}$ phase is also studied. A consistent approach to describing the effect of elastic forces on microstructural evolution is outlined and used to study the kinetics of a multivariant ordering accompanied by alloy decomposition. Our simulations reveal a number of peculiar kinetic features of multivariant orderings, many of them agreeing well with experimental observations.
\end{abstract}

\section{Introduction}

Studies of microstructural evolution under phase transitions of alloy ordering attract great attention from both fundamental and applied points of view; see [1-16] and references therein. There are many theoretical works discussing various aspects of this problem, e.g. [8-16]. However, most of these works [8-14] treat only the simplest B2 ordering with just two types of antiphase-ordered domain (APD) and one type of antiphase boundary (APB) between them. Meanwhile, ordered structures in real alloys are usually much more complex and include many types of APD. For example, under the $\mathrm{DO}_{3}$ ordering on the $\mathrm{BCC}$ lattice there are four types of APD [1], while under the $\mathrm{L}_{2}$ or $\mathrm{L}_{0}$ ordering on the FCC lattice there are four or six types of APD, respectively [6]. In addition, the APDs in the $\mathrm{D}_{3}$ phase may be separated by APBs of two qualitatively different types; see section 4 . Some particular problems of the $\mathrm{L}_{2}$-type orderings were discussed in [15] and [16], but with no general discussion.

The 'multivariant' character of ordering can result in a number of specific kinetic features that are absent for the simplest B2 ordering. These features include, in particular, a possible presence of some transient states under phase transformations, a peculiar topology and alignment of APBs in both transient and stable microstructures, certain specific features of microstructure under alloy decomposition with ordering, particularly when the lattice misfit between the product phases and resulting elastic forces is significant, and other microstructural effects. Most of these effects are mainly related just to the multivariant character of the ordering and are not very sensitive to the details of crystal structure. For example, the presence of four types of APD under both the $\mathrm{DO}_{3}$ and $\mathrm{L}_{2}$ ordering can result in a number of common features of microstructural evolution, even though the underlying crystal lattices, BCC and FCC, are different. 
Keeping in mind these considerations, in the present paper we discuss the kinetic features of multivariant orderings treating as an example the phase transformations involving the formation and evolution of the $\mathrm{D}_{3}$-type-ordered phase. As we are intending to study the effects of 'multivariance' of the ordering, we consider the $\mathrm{D}_{3}$ states in which all four possible types of APD are present in comparable proportions. This may imply, in particular, that these states are evolving from the disordered BCC phase (A2 phase) after a sufficiently rapid quench; such a rapid quench will be simulated below. Note that such $\mathrm{D}_{3}$ states differ from those used in standard experiments with the $\mathrm{D}_{3}$ phase, where a preliminary annealing within the $\mathrm{B} 2$ region of the phase diagram is usually employed and thus only two types of $\mathrm{DO}_{3}$-ordered APD (of a possible four) are present within much larger 'as-quenched' domains which were initially B2 ordered [1-7].

We consider the following phase transformations:

(i) the $\mathrm{A} 2 \rightarrow \mathrm{D}_{3}$ transition between the disordered $\mathrm{A} 2$ phase and the single-phase $\mathrm{D}_{3}$ region of the phase diagram;

(ii) the $\mathrm{A} 2 \rightarrow \mathrm{A} 2+\mathrm{D}_{3}$ transition between the $\mathrm{A} 2$ and the two-phase $\mathrm{A} 2+\mathrm{D} 0_{3}$ region, both with and without a significant elastic interaction;

(iii) the $\mathrm{A} 2 \rightarrow \mathrm{B} 2+\mathrm{D}_{3}$ transition between the $\mathrm{A} 2$ and the two-phase $\mathrm{B} 2+\mathrm{D}_{3}$ region; and

(iv) the $\mathrm{D}_{3} \rightarrow \mathrm{B} 2+\mathrm{D}_{3}$ transition between the $\mathrm{D}_{3}$ and the two-phase $\mathrm{B} 2+\mathrm{D}_{3}$ region.

We employ the master equation approach to the configurational kinetics of non-equilibrium alloys which was described in references [17-20]. Evolution of an alloy in this approach is described by a certain set of exact equations for local concentrations and correlators of their fluctuations. To solve these equations one can use various approximate methods analogous to those employed in the equilibrium statistical physics, such as the kinetic meanfield approximation (KMFA) which was proposed by Gouyet [17] and used in [18-21], the kinetic cluster-field method [19-21] and higher approximations [22].

Since MFA is known to faithfully reproduce all main thermodynamic characteristics of both $\mathrm{B} 2$ and $\mathrm{D}_{3}$ orderings (particularly if the reduced temperature variable $T^{\prime}=T / T_{c}$ is used where $T_{c}$ is the $\mathrm{B} 2$ ordering critical temperature) [23,24], one may assume that the simplest approximation, KMFA, will be sufficient to study the above-mentioned kinetic features. This is also supported by the agreement between the available KMFA results $[12,13,20]$ and those of Monte Carlo simulation of the B2 ordering kinetics [14,25] and the APB structure [24]. It has also been shown that in the studies of the advanced stages of phase transformations (which are considered in this paper) the true vacancy-mediated atomic exchange mechanism can be replaced by an equivalent direct-exchange model [20] which results in a great simplification of the calculations. Therefore, in this paper we employ the KMFA and the direct-exchange model, just as in the previous studies of the B2 ordering [12, 13,20].

When ordering is accompanied by decomposition into phases with a considerable lattice misfit, effects of elastic interaction $v^{e l}$ on microstructural evolution can be important. These effects are usually described with some asymptotic version $[8,9]$ of the full microscopic expression for $v^{e l}$ suggested by Khachaturyan [26]. In this paper we describe a model based on the full expression for $v^{e l}$ and use it to compare the influence of elastic interaction on the kinetics of phase separation under the multivariant $\mathrm{D}_{3}$ ordering with that under the simplest $\mathrm{B} 2$ ordering.

In section 2 we describe the models and methods of simulation employed. Since most of the experimental studies of the $\mathrm{D}_{3}$ ordering were done for the $\mathrm{Fe}-\mathrm{Al}$ - or $\mathrm{Fe}-\mathrm{Si}$-type alloys, we consider two alloy models, 1 and 2, which qualitatively describe the $\mathrm{Fe}-\mathrm{Al}$ and $\mathrm{Fe}-\mathrm{Si}$ alloy systems and correspond to a relatively long-range and a relatively short-range effective pair interaction, respectively. In section 3 we describe the above-mentioned consistent approach to 
the incorporation of the elastic interactions into our kinetic equations. In section 4 we consider the microscopic structure of $\mathrm{APBs}$ in the $\mathrm{DO}_{3}$ phase which helps us to explain many features of the microstructural evolution discussed below. We discuss general features of the distribution of local order parameters and lattice site occupations near various APBs, and also present their analytical description for the case when the $\mathrm{DO}_{3}$ order parameter is small. In section 5 we employ the KMFA to simulate $\mathrm{A} 2 \rightarrow \mathrm{D}_{3}$ transformations for both of our models, 1 and 2 . In section 6 we compare the microstructural evolution in our model 1 after a rapid quench of the initially disordered alloy into the two-phase $\mathrm{A} 2+\mathrm{DO}_{3}$ region with the evolution in a similar model used in [13] after an analogous quench to the $\mathrm{A} 2+\mathrm{B} 2$ region. Here we also compare the influence of the elastic interaction on the microstructural evolution in these two cases. In section 7 we simulate the $\mathrm{A} 2 \rightarrow \mathrm{B} 2+\mathrm{DO}_{3}$ and $\mathrm{DO}_{3} \rightarrow \mathrm{B} 2+\mathrm{DO}_{3}$ transitions in model 2 . Simulations described in sections 5, 6 and 7 reveal a number of interesting microstructural effects, many of them agreeing well with experimental observations. Our main conclusions are summarized in section 8 .

\section{Models and methods of simulation}

Let us first discuss the phenomenological description of the homogeneous $\mathrm{B} 2$ - and $\mathrm{D}_{3}$-ordered structures $[6,26]$. We consider a binary alloy $\mathrm{A}_{c} \mathrm{~B}_{1-c}$ with the mean concentration $c \leqslant 0.5$. In the B2-ordered structure, the concentration $c_{i}$ at site $i$ with the BCC lattice vector $\boldsymbol{R}_{i}$ can be written as

$$
c_{i}=c+\eta \exp \left(\mathrm{ig}_{1} \cdot \boldsymbol{R}_{i}\right)
$$

where $\eta$ is the B2 order parameter, $g_{1}=[111] 2 \pi / a$ is the B2 superstructure vector and $a$ is the BCC lattice constant. Equation (1) shows that under the B2 ordering the BCC lattice splits into two simple cubic sublattices, 1 and 2 , with the concentrations $c_{1}=c+\eta$ and $c_{2}=c-\eta$. The thermodynamic equilibrium conditions determine the value of $|\eta|$, but not the sign of $\eta$, so two types of ordered domain differing in the sign of $\eta$ are possible.

The $\mathrm{DO}_{3}$ structure corresponds to a further ordering of one of the sublattices of the B2 structure and to the presence of two order parameters, $\eta$ and $\zeta$. The concentration at site $i$ can be written as

$$
c_{i}=c+\eta \exp \left(\mathrm{ig}_{1} \cdot \boldsymbol{R}_{i}\right)+2 \zeta\left[\theta(\eta) \cos \left(\boldsymbol{g}_{2} \cdot \boldsymbol{R}_{i}\right)+\theta(-\eta) \sin \left(\boldsymbol{g}_{2} \cdot \boldsymbol{R}_{i}\right)\right]
$$

where $\boldsymbol{g}_{2}=[111] \pi / a$ is the $\mathrm{DO}_{3}$ superstructure vector and $\theta(x)$ is the Heaviside function: $\theta(x)=1$ at $x>0$ and zero otherwise. The structure of the last term in (2) reflects the fact that additional $\mathrm{DO}_{3}$ ordering occurs in the $\mathrm{B} 2$ sublattice which is enriched by the minority component.

It is convenient to describe the $\mathrm{BCC}$ lattice with $\mathrm{DO}_{3}$ ordering with the help of the four interpenetrating FCC sublattices, to be labelled by index $\alpha=$ I, II, III or IV, with lattice parameter $2 a$ and the following coordinates of the basic site: I: (000) $a$; II: (100) $a$; III: (111) $a / 2$;

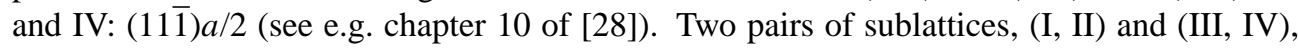
form the two above-mentioned simple cubic lattices 1 and 2.

As the thermodynamic potentials, for symmetry reasons, cannot depend on the signs of $\eta$ and $\zeta$, there are four types of ordered domain differing in these signs. For example, equation (2) gives the following concentrations in the four FCC sublattices for the domain with $\eta>0$ and $\zeta>0$ :

$$
c_{\mathrm{I}}=c+\eta+2 \zeta \quad c_{\mathrm{II}}=c+\eta-2 \zeta \quad c_{\mathrm{III}}=c_{\mathrm{IV}}=c-\eta .
$$

Different APDs are separated by APBs of two different types. The APB separating two APDs differing in the sign of $\eta$ (which implies that $\eta$ vanishes within such an APB) will be 
called below an ' $\eta$-APB', or a B2-type APB [7], while the APB separating two APDs with the same sign of $\eta$ and different signs of $\zeta$ and including the surface $\zeta=0$ will be called a ' $\zeta$-APB', or a $\mathrm{D}_{3}$-type APB [7]. The internal structure of these two types of APB will be described below in section 4 .

In the microscopical description $[19,20]$, various distributions of atoms over lattice sites $i$ are specified using the occupation number operator $n_{i}$, equal to unity when an atom $\mathrm{A}$ is at the site $i$ and zero otherwise. Non-equilibrium alloy states are described in terms of mean occupations $c_{i}=\left\langle n_{i}\right\rangle$ which are averaged over the time-dependent probability distribution of the $n_{i}$-values $[18,19]$. For the not-very-early stages of evolution discussed below when the microstructure is already somewhat 'coarsened' and includes sufficiently large ordered domains, such a description appears to be both complete and consistent [20]. The time evolution of an alloy will be described by the KMFA equation [17] which in the notation used below has the form [19]

$$
\frac{\mathrm{d} c_{i}}{\mathrm{~d} t}=\sum_{j} M_{i j} 2 \sinh \left[\beta\left(F_{j}-F_{i}\right) / 2\right] .
$$

Here: $\beta=1 / T$ is the reciprocal temperature; $F_{i}=\partial F / \partial c_{i}$ is the partial derivative of the free energy of a non-uniform alloy $F\left\{c_{i}\right\}$ with respect to $c_{i}$; and $M_{i j}$ is the generalized mobility. The explicit expressions of $F_{i}$ and $M_{i j}$ for the MFA and the pair interaction model are

$$
\begin{aligned}
& F_{i}=T \ln \frac{c_{i}}{c_{i}^{\prime}}+\sum_{j} v_{i j} c_{j} \\
& M_{i j}^{\mathrm{MFA}}=\gamma_{i j}\left\{c_{i} c_{i}^{\prime} c_{j} c_{j}^{\prime} \exp \left[\beta \sum_{k}\left(u_{i k}+u_{j k}\right) c_{k}\right]\right\}^{1 / 2} .
\end{aligned}
$$

Here: $c_{i}^{\prime}=1-c_{i} ; v_{i j}=V_{i j}^{\mathrm{AA}}+V_{i j}^{\mathrm{BB}}-2 V_{i j}^{\mathrm{AB}}$ is the effective pair interaction between the sites $i$ and $j ; u_{i j}=V_{i j}^{\mathrm{AA}}-V_{i j}^{\mathrm{BB}}$ is the analogous 'asymmetric' pair interaction; and $\gamma_{i j}$ is the configurationally independent factor in the probability of an atomic exchange $\mathrm{A} \leftrightarrow \mathrm{B}$ between the sites $i$ and $j$ per unit time. For simplicity, below we suppose the asymmetric potentials to be zero: $u_{i j}=0$, the intersite atomic jumps to occur only between nearest-neighbour sites: $\gamma_{i j}=\gamma_{n n}$, and we use a 'reduced' time variable $t^{\prime}=t \gamma_{n n}$.

For the effective pair interactions $v_{i j}=v\left(\boldsymbol{R}_{i j}\right)$ we employ two sets of values describing two models, 1 and 2 . Model 1 qualitatively corresponds to the Fe-Al-type alloys. The $v_{i j}$ values for this model were taken from the work of Hasaka [30] where the observed phase diagram of the $\mathrm{Fe}-\mathrm{Al}$ system in the $c, T$-range of our interest was fairly well reproduced by the MFA calculation with the following relations between the interactions $v_{n}$ for first, second and third neighbours:

$$
v_{1}>0 \quad v_{2} / v_{1}=0.184 \quad v_{3} / v_{1}=-0.844 \quad v_{n} \geqslant 4=0 .
$$

The presence of a significant constant $v_{3}$ in (7) implies the interactions in the model 1 to be sufficiently long range. It results, in particular, in a virtual absence of the crystallographic anisotropy effect on microstructural evolution under $\mathrm{D}_{3}$ ordering.

The model 2 qualitatively corresponds to the Fe-Si-type alloys. The $v_{n}$-values for this model were taken from the MFA calculations of Inden [31] which satisfactorily describe the relevant part of the observed $\mathrm{Fe}-\mathrm{Si}$ phase diagram $[4,32]$ with the following relations [33]:

$$
v_{1}>0 \quad v_{2} / v_{1}=0.5 \quad v_{n} \geqslant 3=0 .
$$

The last equation (8) implies the interactions in the model 2 to be relatively short range. This results in a number of microstructural features under $\mathrm{D}_{3}$ ordering - in particular, in strong crystallographic alignment of some APBs discussed in section 5. 
The MFA phase diagrams for both of our alloy models are presented in figure 1 where we also indicate the alloy states chosen for the computer simulations described below.
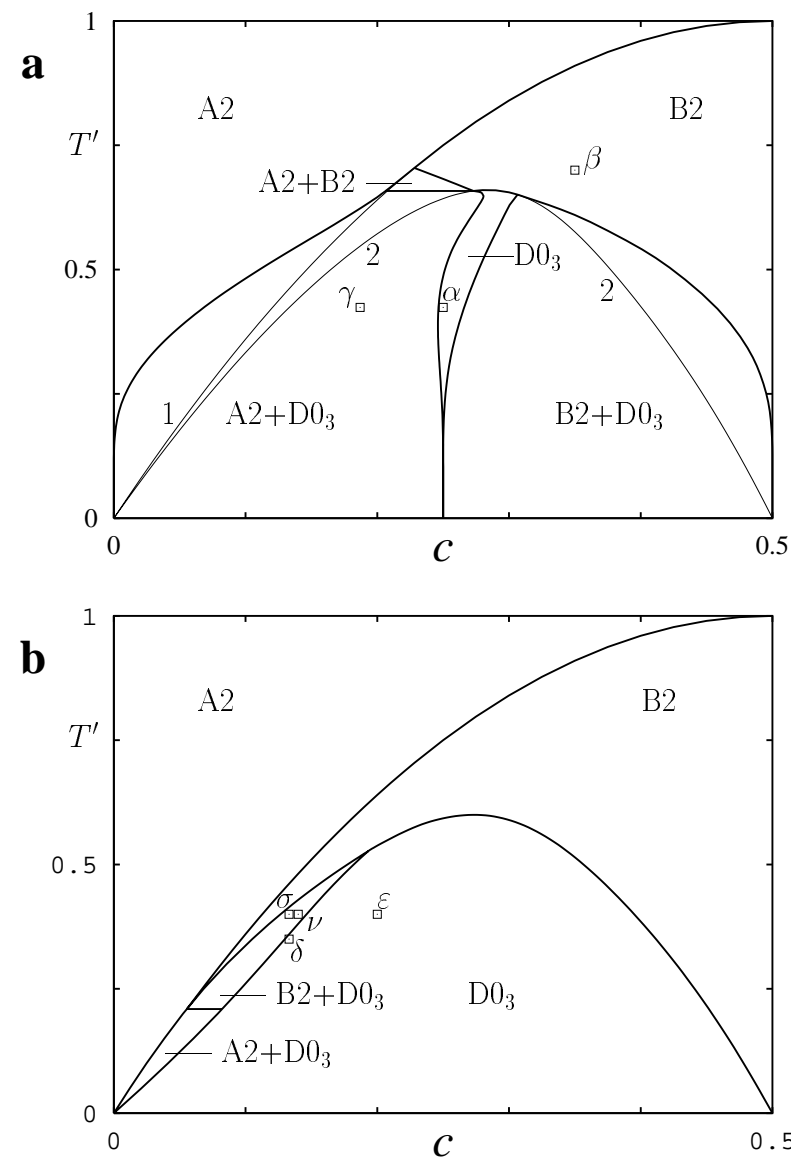

Figure 1. (a) The phase diagram for the Fe-Al-type model 1 described in the text. Curve 1 is the $\mathrm{B} 2$-ordering spinodal and curve 2 is the $\mathrm{D}_{3}$-ordering spinodal. (b) The phase diagram for the Fe-Si-type model 2 described in the text.

To describe the inhomogeneous states of a partially ordered alloy, in particular the APBs, it is convenient to define 'local order parameters' which correspond to a spatial averaging over some local region. This region can correspond, for example, to some extended crystal cell including several lattice sites $[13,27]$. For the $\mathrm{D}_{3}$ ordering such an extended cell can be chosen as the elementary cell of the FCC lattice with the parameter $2 a$ which includes four sites belonging to the four above-mentioned FCC sublattices $\alpha$. Then the local order parameters $\eta_{k}, \zeta_{1 k}, \zeta_{2 k}$ and the local mean concentration $\bar{c}_{k}$ for the extended cell $k$ are related to the site occupations $c_{i}=c_{k \alpha}$ as follows:

$$
c_{k \alpha}=\bar{c}_{k}+\eta_{k} \exp \left(\mathrm{i} \boldsymbol{g}_{1} \cdot \boldsymbol{R}_{k \alpha}\right)+2 \zeta_{1 k} \cos \left(\boldsymbol{g}_{2} \cdot \boldsymbol{R}_{k \alpha}\right)+2 \zeta_{2 k} \sin \left(\boldsymbol{g}_{2} \cdot \boldsymbol{R}_{k \alpha}\right)
$$

where $\boldsymbol{R}_{k \alpha}$ is the lattice vector for site $k \alpha$. In a homogeneous state the local order parameters in all cells $k$ are the same and are connected with occupations $c_{\alpha}$ of the four FCC sublattices $\alpha$ as follows:

$$
\eta=\left(c_{\mathrm{I}}+c_{\mathrm{II}}-c_{\mathrm{III}}-c_{\mathrm{IV}}\right) / 4 \quad \zeta_{1}=\left(c_{\mathrm{I}}-c_{\mathrm{II}}\right) / 4 \quad \zeta_{2}=\left(c_{\mathrm{IV}}-c_{\mathrm{III}}\right) / 4
$$


and according to equation (2) either $\zeta_{2}=0$ (for $\eta>0$ ) or $\zeta_{1}=0$ (for $\eta<0$ ).

The 'extended-cell' averaging used in (9) needs some specific choice of these cells. Therefore, it can be unsuitable for describing an arbitrarily inhomogeneous local order. A more convenient description is usually provided by the 'site-centred' local order parameters for which the averaging is taken over some nearest neighbourhood of each site $i$, while its occupation $c_{i}$ makes the largest contribution to the averages $[12,20]$. For the $\mathrm{D}_{3}$ ordering the site local order parameter $\eta_{i}$ and the site mean concentration $\bar{c}_{i}$ can be defined similarly to those for the B2 phase [20]:

$$
\begin{aligned}
\eta_{i} & =\frac{1}{4}\left(c_{i}-\frac{2}{z_{n n}} \sum_{j=n n(i)} c_{j}+\frac{1}{z_{n n n}} \sum_{j=n n n(i)} c_{j}\right) \\
\bar{c}_{i} & =\frac{1}{4}\left(c_{i}+\frac{2}{z_{n n}} \sum_{j=n n(i)} c_{j}+\frac{1}{z_{n n n}} \sum_{j=n n n(i)} c_{j}\right)
\end{aligned}
$$

where $n n(i)$ and $n n n(i)$ indicate summation over nearest and next-nearest neighbours of site $i$, while $z_{n n}$ and $z_{n n n}$ are the total numbers of such neighbours.

The local $\zeta$-type order is conveniently characterized by the quantity $\zeta_{i}^{2}=\zeta_{1 i}^{2}+\zeta_{2 i}^{2}$ where $\zeta_{1 i}$ and $\zeta_{2 i}$ are 'site-centred' analogues of $\zeta_{1 k}$ and $\zeta_{2 k}$ in (9). Then the site order parameter $\zeta_{i}$ can be defined by the relation

$$
\zeta_{i}^{2}=\frac{1}{16}\left[\left(c_{i}-\frac{1}{z_{n n n}} \sum_{n n n(i)} c_{j}\right)^{2}+\left(\frac{2}{z_{n n}} \sum_{n n(i)} \sin \left(\boldsymbol{g}_{2} \cdot \boldsymbol{R}_{i j}\right) c_{j}\right)^{2}\right]
$$

where $\boldsymbol{R}_{i j}$ is $\boldsymbol{R}_{j}-\boldsymbol{R}_{i}$, and the coefficient 1/16 on the right-hand side was chosen so that in the homogeneous case (2) we would have $\zeta_{i}^{2}=\zeta^{2}$.

From the experimental point of view, the description of alloy states in terms of local concentrations $c_{i}$ corresponds to the high-resolution electron microscopy (HREM) images where the occupations of individual lattice sites (averaged over atomic columns of the extinction length scale) are displayed. This ' $c_{i}$-representation' is convenient for describing the atomicscale microstructures characteristic for the initial stages of phase transformations discussed below. Later stages correspond to the formation of more or less extended ordered regions which are more conveniently described by local order parameter distributions. Such distributions are experimentally observed in the diffraction transmission electron microscopy (TEM) images where the reflection intensity for a certain superstructure vector is proportional to the squared value of the relevant order parameter [1-7]. In particular, for the vector $g_{1}$ defined in equation (1) the reflection intensity for the homogeneous $\mathrm{D}_{3}$ phase is proportional to $\eta^{2}$ and vanishes at $\eta$-APBs, while for the vector $\boldsymbol{g}_{2}$ in equation (2) this intensity for the homogeneous $\mathrm{D}_{3}$ phase is proportional to $\zeta^{2}$ and vanishes both at $\zeta$-APBs and $\eta$-APBs [6]. Thus the distribution of the reflection intensities over an inhomogeneous alloy is similar to that of the local order parameters $\eta_{i}^{2}$ or $\zeta_{i}^{2}$. Therefore, the latter distributions (to be called below $\eta^{2}$ and $\zeta^{2}$-representations, respectively) can be directly compared with the experimental TEM images.

For our simulations we use both three-dimensional and two-dimensional (3D and 2D) lattice models. Employing 2D models enables one to significantly extend the maximum size of microstructures examined which makes the simulation much more informative. Moreover, simulation of a $2 \mathrm{D}$ version of the $\mathrm{D}_{3}$ ordering allows us to study also a kinetic behaviour of the so-called 'conservative' APBs which are often observed under multivariant orderings, e.g. under the $\mathrm{L}_{2}$ and $\mathrm{L}_{0}$ orderings [6,34]. For geometrical reasons discussed below, the conservative APBs are not formed in realistic $3 \mathrm{D}$ models of $\mathrm{D} 0_{3}$ ordering, such as our models 1 and 2, but such APBs can arise in a 2D version of model 2. The 2D analogue of 
the BCC lattice is just a simple square lattice. The superstructure vector $\boldsymbol{g}_{1}$ in equation (1) for the 2D lattice is [11] $\pi / a$, while in equation (2) the factor $2 \cos \left(\boldsymbol{g}_{2} \cdot \boldsymbol{R}_{i}\right)$ is replaced by the sum $\cos \left(\boldsymbol{g}_{2 x} \cdot \boldsymbol{R}_{i}\right)+\cos \left(\boldsymbol{g}_{2 y} \cdot \boldsymbol{R}_{i}\right)$ and the factor $2 \sin \left(\boldsymbol{g}_{2} \cdot \boldsymbol{R}_{i}\right)$ is replaced by the difference $\cos \left(\boldsymbol{g}_{2 x} \cdot \boldsymbol{R}_{i}\right)-\cos \left(\boldsymbol{g}_{2 y} \cdot \boldsymbol{R}_{i}\right)$, where $\boldsymbol{g}_{2 x}=[10] \pi / a, \boldsymbol{g}_{2 y}=[01] \pi / a$ and $a$ is the square lattice constant. The four FCC sublattices of the 3D BCC lattice then correspond to the four simple quadratic sublattices with lattice constant $2 a$ and the following coordinates of the basic site: I: (00) $a$; II: (11) $a$; III: (10) $a$; IV: (01) $a$. With these changes of notation, relations (1)-(3) and their structural implications hold for both the $3 \mathrm{D}$ case and the $2 \mathrm{D}$ case. Therefore, simulation on the $2 \mathrm{D}$ lattice can be conveniently used to study the kinetics of the $\mathrm{D}_{3}$-type orderings, just as for the B2-type orderings [8,9, 12-14].

In the treatments of $2 \mathrm{D}$ versions of our alloy models 1 and 2 we chose the effective pair interactions such that their MFA phase diagrams were identical to those for the 3D models shown in figure 1. The thermodynamic formulae of the MFA corresponding to the ordered phase described by concentration waves with the wave vectors $\boldsymbol{k}_{m}$ (which include the superstructure vectors $\boldsymbol{k}_{s}$ and $\boldsymbol{k}=0$ ) include the interaction $v(\boldsymbol{R})$ only via its Fourier components $v(\boldsymbol{k})$ at $\boldsymbol{k}=\boldsymbol{k}_{m}$; see e.g. [26,30,31]. Hence the MFA phase diagrams with A2, $\mathrm{B} 2$ and $\mathrm{DO}_{3}$ phases are determined by just three such Fourier components, $v(0), v\left(g_{1}\right)$ and $v\left(\boldsymbol{g}_{2}\right)$ (or $v(0), v\left(\boldsymbol{g}_{1}\right)$ and $v\left(\boldsymbol{g}_{2 x}\right)$ in the 2D case), where $\boldsymbol{g}_{1}$ and $\boldsymbol{g}_{2}$ or $\boldsymbol{g}_{2 x}$ are the above-discussed superstructure vectors for the $\mathrm{B} 2$ and $\mathrm{DO}_{3}$ phase in the $3 \mathrm{D}$ or $2 \mathrm{D}$ lattice. Equating each of these $v\left(\boldsymbol{k}_{m}\right)$ for the 3D model to $v\left(\boldsymbol{k}_{m}\right)$ for the 2D model we make the MFA phase diagrams of the two models identical. For the two- or three-neighbour interaction models considered, these equations yield the following relations between interaction constants $v_{n}$ in the $2 \mathrm{D}$ and 3D models:

$$
\left(v_{2} / v_{1}\right)_{2 \mathrm{D}}=0.75\left(v_{2} / v_{1}\right)_{3 \mathrm{D}} \quad\left(v_{3} / v_{1}\right)_{2 \mathrm{D}}=1.5\left(v_{3} / v_{1}\right)_{3 \mathrm{D}}
$$

where $\left(v_{n} / v_{1}\right)_{3 \mathrm{D}}$ is the value of $v_{n} / v_{1}$ in equation (7) or (8).

The 2 D simulations were performed on a square lattice of $192 \times 192$ or $128 \times 128$ sites and the $3 \mathrm{D}$ simulation was performed on a BCC lattice of $40^{3} \times 2$ sites, with periodic boundary conditions in all cases. The simulation methods were the same as in references $[13,18,20]$. In simulations of the $\mathrm{A} 2 \rightarrow \mathrm{DO}_{3}, \mathrm{~A} 2 \rightarrow \mathrm{A} 2+\mathrm{DO}_{3}$ and $\mathrm{A} 2 \rightarrow \mathrm{B} 2+\mathrm{DO}_{3}$ transformations the initial as-quenched distribution $c_{i}(0)$ was characterized by its mean value $c$ and small random fluctuations $\delta c_{i}$; usually we used $\delta c_{i}= \pm 0.01$.

\section{Description of elastic interactions}

A consistent model for describing effective pair interactions $v^{e l}$ due to elastic forces in a dilute alloy has been proposed by Khachaturyan [26] (see also [35]). However, in applications to alloy kinetics $[8-11,36]$ Khachaturyan and co-workers used only some asymptotic form of this $v^{e l}$ whose accuracy was not studied. In this section we describe a model of $v^{e l}$ which is based on the full microscopic expressions of references [26,35] and is used in the simulations of phase separation with ordering described in section 6 .

The approach $[26,35]$ is based on the expression for the full Hamiltonian $H$ of a dilute alloy as a function of occupation numbers $n_{i}$ and atomic displacements $\boldsymbol{u}_{i}$. It includes both the standard quadratic-in- $\boldsymbol{u}_{i}$ term describing the harmonic oscillations of atoms (phonons) and the so-called Kanzaki term linear in both $\boldsymbol{u}_{i}$ and $n_{i}$ :

$$
H=H_{c}\left\{n_{i}\right\}+\frac{1}{2} \sum_{i j} A_{i j}^{\alpha \beta} u_{i}^{\alpha} u_{j}^{\beta}-\sum_{i j} \boldsymbol{F}_{i j} \cdot \boldsymbol{u}_{i} n_{j} .
$$

Here: $H_{c}$ is the energy of an alloy with all $\boldsymbol{u}_{i}$ equal to zero (the 'chemical', or 'short-range' energy); $A_{i j}^{\alpha \beta}$ is the matrix of force constants; the parameters $\boldsymbol{F}_{i j}$ describe the 'forces' exerted by 
the impurity atom on the adjacent main-component atoms; and the summation over repeated Greek indices is implied. The force constant matrix $A_{i j}^{\alpha \beta}=A^{\alpha \beta}\left(\boldsymbol{R}_{i}-\boldsymbol{R}_{j}\right)$ is supposed to correspond to the pure main component and thus does not depend on local occupations (the 'dilute-alloy' assumption). This assumption excludes from consideration, in particular, the effects connected with the difference of the elastic constants in different phases which can be important for some problems.

To find the equilibrium values of $\boldsymbol{u}_{i}$ at given $n_{i}$ one should minimize the expression (15) with respect to $\boldsymbol{u}_{i}$. This yields the relation

$$
D_{\alpha \beta}(\boldsymbol{k}) u_{\beta}(\boldsymbol{k})=F_{\alpha}(\boldsymbol{k}) n(\boldsymbol{k})
$$

where $D_{\alpha \beta}(\boldsymbol{k}), \boldsymbol{F}(\boldsymbol{k})$ and $n(\boldsymbol{k})$ are the Fourier components of $A^{\alpha \beta}\left(\boldsymbol{R}_{i j}\right), \boldsymbol{F}\left(\boldsymbol{R}_{i j}\right)$ and $n_{i}=$ $n\left(\boldsymbol{R}_{i}\right)$, respectively. Introducing the tensor $G_{\alpha \beta}(\boldsymbol{k})$ inverse to the dynamical matrix $D_{\alpha \beta}(\boldsymbol{k})$ : $G_{\alpha \beta}(\boldsymbol{k}) D_{\beta \gamma}(\boldsymbol{k})=\delta_{\alpha \gamma}$, we can express $\boldsymbol{u}(\boldsymbol{k})$ via $n(\boldsymbol{k})$ :

$$
u_{\alpha}(\boldsymbol{k})=G_{\alpha \beta}(\boldsymbol{k}) F_{\beta}(\boldsymbol{k}) n(\boldsymbol{k}) .
$$

Substituting this expression for $\boldsymbol{u}(\boldsymbol{k})$ into (15) and supposing for simplicity that the shortrange term $H_{c}$ includes only pairwise interactions $v_{i j}^{c}=v^{c}\left(\boldsymbol{R}_{i j}\right)$, we obtain [26]

$$
H=\frac{1}{2} \sum_{i j}\left(v_{i j}^{c}+v_{i j}^{e l}\right) n_{i} n_{j}
$$

where the Fourier component of the elastic interaction $v_{i j}^{e l}=v^{e l}\left(\boldsymbol{R}_{i j}\right)$ is

$$
v^{e l}(\boldsymbol{k})=-F_{\alpha}(\boldsymbol{k}) G_{\alpha \beta}(\boldsymbol{k}) F_{\beta}^{*}(\boldsymbol{k}) .
$$

Let us mention that the elastic interaction $v_{i j}^{e l}$ in (18) with $v^{e l}(\boldsymbol{k})$ defined by equation (19) does not vanish at $i=j$ (unlike the chemical interaction $v_{i j}^{c}$ ), but due to the identity $n_{i}^{2}=n_{i}$ it yields just a constant term

$$
v_{i i}^{e l} \sum_{i} n_{i}=v_{i i}^{e l} N_{A}
$$

in energy (18). As was discussed in detail by Khachaturyan [26], this term (as well as other similar terms omitted in our equation (15)) describes a 'configurationally independent' contribution to the energy which does not affect the evolution of inhomogeneous atomic distributions being studied in this work.

In this paper we consider the elastic interaction for the $2 \mathrm{D}$ version of our Fe-Al-type model 1. For simplicity we assume that the force constants $A_{\alpha \beta}\left(\boldsymbol{R}_{i j}\right)$ are non-zero only for the first and second neighbours, and that for the second neighbours they correspond to the spherically symmetrical interaction

$$
A_{x x}(1,1)=A_{x y}(1,1)=A_{y y}(1,1)
$$

where the components of $\boldsymbol{R}$ are given in units of the lattice constant $a$.

For this model we can express the dynamical matrix $\boldsymbol{D}(\boldsymbol{k})$ via the experimental elastic constants $c_{i k}$, comparing its long-wavelength limit with the relevant formulae of the elasticity theory. This results in the following expressions for $D_{\alpha \beta}(k)$ :

$$
\begin{gathered}
D_{x x}(\boldsymbol{k})=4\left\{A_{x x}(1,0) \sin ^{2}\left(k_{x} a / 2\right)+A_{x x}(0,1) \sin ^{2}\left(k_{y} a / 2\right)\right. \\
\left.+A_{x x}(1,1)\left[1-\cos \left(k_{x} a\right) \cos \left(k_{y} a\right)\right]\right\} \\
\begin{array}{c}
D_{y y}(\boldsymbol{k})=4\left\{A_{y y}(1,0) \sin ^{2}\left(k_{x} a / 2\right)+A_{y y}(0,1) \sin ^{2}\left(k_{y} a / 2\right)\right. \\
\left.+A_{y y}(1,1)\left[1-\cos \left(k_{x} a\right) \cos \left(k_{y} a\right)\right]\right\}
\end{array} \\
D_{x y}(\boldsymbol{k})=4 A_{x y}(1,1) \sin \left(k_{x} a\right) \sin \left(k_{y} a\right)
\end{gathered}
$$


where

$$
\begin{aligned}
& A_{x x}(1,0)=A_{y y}(0,1)=c_{11}-\left(c_{44}+c_{12}\right) / 2 \\
& A_{x x}(0,1)=A_{y y}(1,0)=\left(c_{44}-c_{12}\right) / 2 \\
& A_{x x}(1,1)=A_{y y}(1,1)=A_{x y}(1,1)=\left(c_{44}+c_{12}\right) / 4 .
\end{aligned}
$$

The values of $F_{\alpha}(\boldsymbol{k})$ for an alloy $\mathrm{A}_{c} \mathrm{~B}_{1-c}$ can be expressed via the observable concentrational expansion coefficient $u_{0}=\mathrm{d} \ln a / \mathrm{d} c$ if one assumes that the $F_{\alpha}(\boldsymbol{R})$ are nonzero only for nearest neighbours [26] (this assumption may lead to some quantitative errors but seems to be adequate for making estimates [37]). Then $F_{\alpha}(\boldsymbol{k})$ takes the form

$$
F_{\alpha}(\boldsymbol{k})=-2 \mathrm{i} a B u_{0} \sin \left(k_{\alpha} a\right)
$$

where $B=\left(c_{11}+c_{12}\right) / 2$ is the bulk modulus in the $2 \mathrm{D}$ case.

Substituting the values of $D_{\alpha \beta}(\boldsymbol{k})$ and $F_{\alpha}(\boldsymbol{k})$ in equation (19) we obtain the elastic potential $v^{e l}$. In particular, the asymptotic form of $v^{e l}(\boldsymbol{k})$ at small $k$ determining the long-range behaviour of the elastic interaction is

$$
v_{a s}^{e l}(\boldsymbol{k})=-4 \Omega B^{2} u_{0}^{2} \frac{1+2 \Delta n_{x}^{2} n_{y}^{2}}{c_{11}+\left(c_{11}+c_{12}\right) \Delta n_{x}^{2} n_{y}^{2}}
$$

where: $\Omega=a^{2}$ is the elementary-cell area; $n_{\alpha}$ is $k_{\alpha} / k$; and the parameter

$$
\Delta=\left(c_{11}-c_{12}-2 c_{44}\right) / c_{44}
$$

characterizes the elastic anisotropy. The expression (28) is similar to its $3 \mathrm{D}$ analogue (see e.g. [38]):

$v_{a s, 3 \mathrm{D}}^{e l}(\boldsymbol{k})=-\frac{9 \Omega B^{2} u_{0}^{2}\left[1+2 \Delta\left(n_{x}^{2} n_{y}^{2}+n_{y}^{2} n_{z}^{2}+n_{z}^{2} n_{x}^{2}\right)+3 \Delta^{2} n_{x}^{2} n_{y}^{2} n_{z}^{2}\right]}{c_{11}+\left(c_{11}+c_{12}\right) \Delta\left(n_{x}^{2} n_{y}^{2}+n_{y}^{2} n_{z}^{2}+n_{z}^{2} n_{x}^{2}\right)+\Delta^{2}\left(c_{11}+2 c_{12}+c_{44}\right) n_{x}^{2} n_{y}^{2} n_{z}^{2}}$

where the bulk modulus $B$ is $\left(c_{11}+2 c_{12}\right) / 3$ and $\Omega$ is the elementary-cell volume, i.e. $a^{3} / 2$ or $a^{3} / 4$ for the BCC or FCC lattice, respectively.

The anisotropy of the elastic interaction can be characterized by the difference $v_{a n}^{e l}=$ $v_{a s}^{e l}\left(\boldsymbol{n}_{m}\right)-v_{a s}^{e l}\left(\boldsymbol{n}_{0}\right)$ where $\boldsymbol{n}_{m}$ and $\boldsymbol{n}_{0}$ correspond to the directions of maximum and minimum of $v_{a s}^{e l}(\boldsymbol{n})$, respectively; for the usual case $\Delta<0$, the vector $\boldsymbol{n}_{m}$ is $2^{-1 / 2}[1,1]$ and $\boldsymbol{n}_{0}$ is [1,0]. The importance of this anisotropy can be estimated as the ratio $v_{a n}^{e l} / T_{c}$ where $T_{c}$ is the critical ordering temperature. For estimates, the difference $v_{a n}^{e l}$ can be found using only the first-order term in the Taylor expansion of the expression (28) or (29) in powers of $\Delta$, while for $T_{c}$ we can use the MFA expression $\left[-v\left(\boldsymbol{k}_{s}\right)\right] / 4$ where $v=v^{c}+v^{e l}$ is the full interaction potential and $\boldsymbol{k}_{s}$ is the ordering superstructure vector. Then the importance of the elastic effects for microstructural evolution can be characterized by the dimensionless parameter

$$
\lambda=w /\left|v^{c}\left(\boldsymbol{k}_{s}\right)+v^{e l}\left(\boldsymbol{k}_{s}\right)\right|
$$

where, for our 2D case (28), $w=4 a^{2} B^{2} u_{0}^{2}\left(c_{11}-c_{12}\right)(-\Delta) / c_{11}^{2}$, and in studies of both B2 and $\mathrm{DO}_{3}$ ordering we put $\boldsymbol{k}_{s}=\boldsymbol{g}_{1}$.

\section{Structure of antiphase boundaries in the $\mathrm{DO}_{3}$ phase}

In this section we discuss the microscopical structure of the two types of APB in the $\mathrm{D}_{3}$ phase. This discussion has its own interest—see e.g. [6,7,29]—and it also can help us to understand the kinetic features of $\mathrm{DO}_{3}$ ordering discussed below.

The APB of the first type is a $\zeta$-APB separating two APDs with the same sign of $\eta$ and different signs of $\zeta_{1}$ or $\zeta_{2}$ (whichever is non-zero). The APB of the second type is an $\eta$-APB 
separating two APDs with different signs of $\eta$ which implies vanishing of $\eta$ within the APB. In other words, on this APB the two above-mentioned pairs of sublattices $\alpha$, (I, II) and (III, IV), 'exchange their places'. If an alloy is quenched from the $\mathrm{B} 2$ state to the $\mathrm{D}_{3}$ region of the phase diagram, the 'inherited' APBs from the B2 state become the $\eta$-APBs, while the newly formed $\mathrm{D}_{3}$ domains form the new $\zeta$-APBs. The kinetics of the $\mathrm{D}_{3}$ ordering of a singledomain (annealed) B2 alloy is therefore quite similar to the extensively studied B2 ordering of an initially disordered alloy [8-14] and thus is not of interest for the present research on multivariant orderings.

First, we derive the analytical expressions for local order parameters and mean occupations near a $\zeta$-APB for the $c, T$-range just below the $\mathrm{D}_{3}$-ordering spinodal where the equilibrium order parameter $\zeta_{0}=\zeta_{0}(c, T)$ is small. The structure of an $\eta$-APB in this region does not significantly change with respect to that in the $\mathrm{B} 2$ phase: the corrections to the $c_{i}$ - and $\eta_{i}$-values due to 'weak' $\mathrm{D}_{3}$ ordering are small compared to their own variations near this $\eta$-APB where $\eta_{i}$-values (being generally not small) even change their sign.

The $\mathrm{D}_{3}$ spinodal line $T=T_{s}(c)$ (which in the case of the second-order $\mathrm{B} 2 \leftrightarrow \mathrm{D}_{3}$ transition coincides with the phase boundary between these two phases) in the MFA used is defined by the following equation:

$$
c_{+}(c) c_{+}^{\prime}(c)=-T_{s}(c) / v\left(\boldsymbol{g}_{2}\right) .
$$

Here $v\left(g_{2}\right)$ is the Fourier component of the interaction $v_{i j}$ at $\boldsymbol{k}=\boldsymbol{g}_{2}, c_{+}=c+\eta_{0}$ and $c_{+}^{\prime}=1-c_{+}$, where $c$ is the mean concentration and $\eta_{0}=\eta_{0}(c, T)$ is the equilibrium $\eta$-value for the given $c$ and $T$.

To find the values of $c_{i}, \eta_{i}$ and $\zeta_{i}$ near a $\zeta$-APB we should solve the stationarity equations $F_{i}=\partial F / \partial c_{i}=$ constant [18-20] with boundary conditions $\zeta_{i} \rightarrow \pm \zeta_{0}$ at $\pm \infty$ (we omit the index 1 or 2 of the non-zero $\zeta$-parameter). Near the $\mathrm{D}_{3}$ spinodal curve the solution can be obtained analytically using the expansions in the powers of $\zeta_{0}$, just as for $\eta$-APB near the B2-ordering spinodal [12]. Considering for simplicity the APB lying in the (100) plane and denoting the distance of site $i$ from the APB as $x_{i}$, we can write the resulting expressions for $\zeta_{i}, \eta_{i}$ and $\bar{c}_{i}$ as

$$
\begin{aligned}
& \zeta_{i}=\zeta_{0} \tanh \left(x_{i} / \delta\right) \\
& \eta_{i}-\eta_{0}=\frac{4\left(c_{+}-1 / 2\right)\left[1+\beta v(0) c_{-} c_{-}^{\prime}\right]}{D} \frac{\zeta_{0}^{2}}{\cosh ^{2}\left(x_{i} / \delta\right)} \\
& \bar{c}_{i}-c=\frac{4\left(c_{+}-1 / 2\right)\left[1+\beta v\left(\boldsymbol{g}_{1}\right) c_{-} c_{-}^{\prime}\right]}{D} \frac{\zeta_{0}^{2}}{\cosh ^{2}\left(x_{i} / \delta\right)} .
\end{aligned}
$$

Here $c_{-}=c-\eta_{0}, c_{-}^{\prime}=1-c_{-}$and

$D=c_{+} c_{+}^{\prime}\left\{2+\left[\beta v(0)+\beta v\left(g_{1}\right)\right]\left(c_{+} c_{+}^{\prime}+c_{-} c_{-}^{\prime}\right)+2 \beta v(0) \beta v\left(g_{1}\right) c_{+} c_{+}^{\prime} c_{-} c_{-}^{\prime}\right\}$

while the APB width $\delta$ is related to the effective interaction length $l_{\text {int }}$ defined by the relation

$$
l_{i n t}^{2}=\sum_{j} R_{i j}^{2} v_{i j} \exp \left(\mathrm{ig}_{2} \cdot \boldsymbol{R}_{i j}\right) / 3 v\left(\boldsymbol{g}_{2}\right)
$$

as follows:

$$
\delta=l_{\text {int }} / \sqrt{\tau\left(1+3 G c_{+}\right)}
$$

where $\tau=\left[T_{s}(c)-T\right] / T_{s}(c)$ and $G=\left\{2+\left[\beta v\left(g_{1}\right)+\beta v(0)\right] c_{-} c_{-}^{\prime}\right\} / D$.

The factors $\left[1+\beta v(0) c_{-} c_{-}^{\prime}\right],\left[1+\beta v\left(g_{1}\right) c_{-} c_{-}^{\prime}\right]$ and $D$ in equations (33) and (34) in our models are positive at $T=T_{s}(c)$ (which probably follows from considerations of the thermodynamic stability). Therefore, these equations show that both the local order parameter 
$\eta_{i}$ and the local mean concentration $\bar{c}_{i}$ have either a pit or a hump at the $\zeta$-APB depending on the sign of the difference $c_{+}-1 / 2$. This is a general feature of a $\zeta$-APB in the $\mathrm{DO}_{3}$ phase which is not connected with the smallness of $\zeta_{0}$. Indeed, expanding the MFA equations of stationarity, $F_{i}=$ constant, in powers of $\delta \bar{c}_{i}=\bar{c}_{i}-c, \delta \eta_{i}=\eta_{i}-\eta_{0}$ and $c_{+}-1 / 2$, one can see that at small $c_{+}-1 / 2$ the differences $\delta \bar{c}_{i}$ and $\delta \eta_{i}$ are proportional to $\left(c_{+}-1 / 2\right)$. Therefore, to the left or to the right of the curve $c_{+}(c, T)=1 / 2, \bar{c}_{i}$ and $\eta_{i}$ at the $\zeta$-APB have a pit or a hump, respectively. Let us also note that according to equation (31) the curve $c_{+}=1 / 2$ crosses the $\mathrm{DO}_{3}$ spinodal $T=T_{S}(c)$ at the point of its maximum.

Analytical expressions (32)-(34) describe the $\zeta$-APBs only near the $\mathrm{D}_{3}$-ordering spinodal. At lower temperatures where $\zeta_{0}$ is not small the distribution of occupations $c_{i}$ near an APB may only be calculated numerically. However, to qualitatively understand the character of the variation of $\bar{c}_{i}$ and $\eta_{i}$ in this region, one can suppose (as in the analogous discussion of the variation of $\bar{c}_{i}$ near an $\eta$-APB in the B2 phase [12]) that the $\zeta_{i}$ - and $\eta_{i}$-values approximately obey the 'local equilibrium' relations: $\zeta_{i} \approx \zeta_{0}\left(\bar{c}_{i}, T\right) ; \eta_{i} \approx \eta_{0}\left(\bar{c}_{i}, T\right)$. This assumption implies neglecting the gradient terms which are actually present in our inhomogeneous problem, but they hardly affect the qualitative trends imposed by this 'local equation of state'. Then one may expect that near a $\zeta$-APB where the $\zeta_{i}$ vanish, the local $\bar{c}_{i}$ should approach the 'nearest' $c$-value in the $c, T$-plane for which $\zeta_{0}(c, T)=0$, i.e. its value on the nearest branch of the $\mathrm{DO}_{3}$ spinodal $T_{s}(c)$ for the given temperature. Therefore, to the left of the $T_{s}(c)$ maximum point we can generally expect a pit (and to the right, a hump) of $\bar{c}_{i}$ and $\eta_{i}$ at the $\zeta$-APB.

In figures 2 and 3 we present the profiles of $\bar{c}_{i}, \eta_{i}, \zeta_{i}^{2}$ and concentrations $c_{\alpha i}$ in the FCC sublattices $\alpha$ near a $\zeta$-APB and near an $\eta$-APB, respectively, which have been numerically calculated for our Fe-Al-type model 1 at $c=0.25$ and $T^{\prime}=0.424$ (point $\alpha$ in figure 1(a)). Both APBs are oriented along the (100) plane, and all local order parameters and local mean concentrations are averaged (over their minor variations within the extended lattice cells) in the planes parallel to the APB.

As one can see from figure 2, the pit of $\eta_{i}$ near the $\zeta$-APB is rather small, i.e. the B2-type

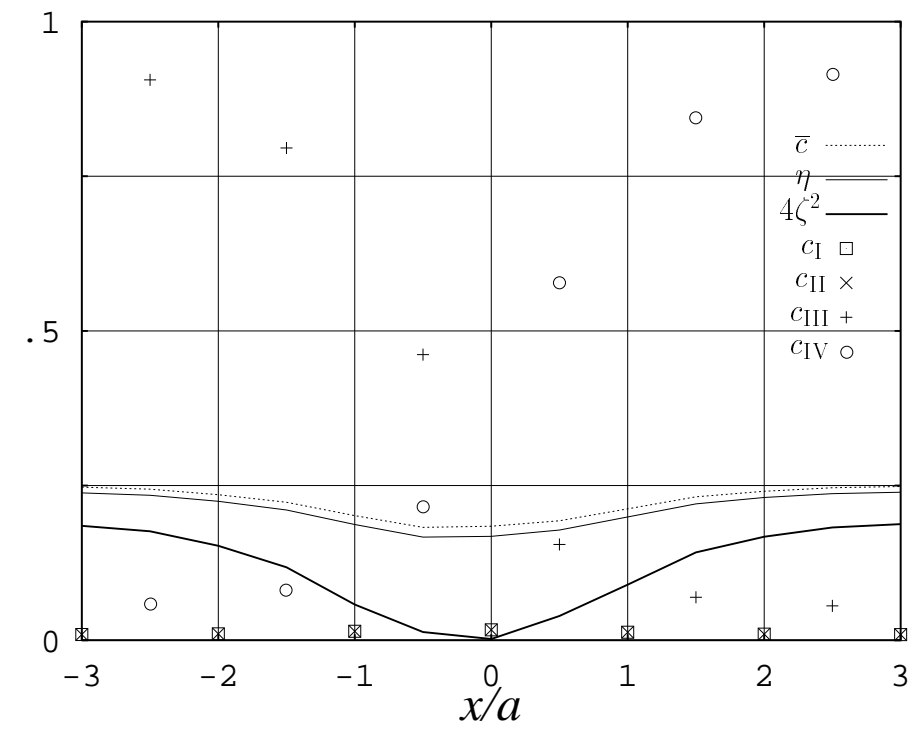

Figure 2. Profiles of the quantities $\bar{c}_{i}, \eta_{i}, 4 \zeta_{i}^{2}$ and $c_{\alpha}$ near a $\zeta$-APB lying in the (100) plane for model 1 at $c=0.25$ and $T^{\prime}=0.424$. 


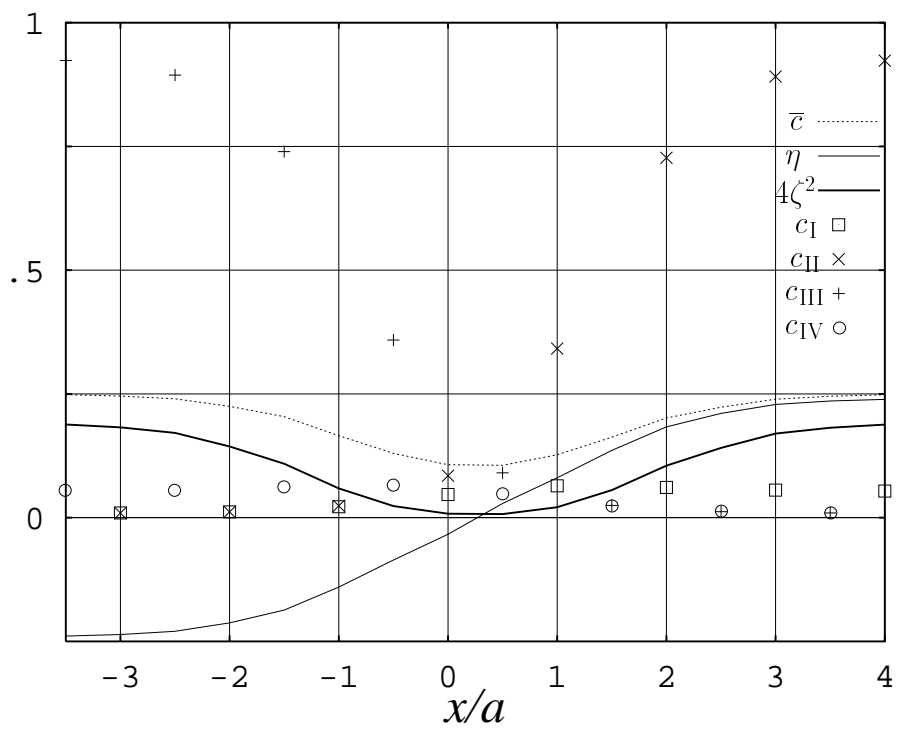

Figure 3. As figure 2, but for an $\eta$-APB.

order is not strongly suppressed at the APB. Therefore, the TEM diffraction contrast on such APBs in the B2 superstructure reflections, e.g. [111], should be much less pronounced compared to that for the $\eta$-APBs where $\eta_{i}$ even vanishes within the boundary. This smallness of the $\eta_{i}$-pit near a $\zeta$-APB is due to the fact that the point considered in the phase diagram is close to the $c_{+}(T)=0.5$ curve where $\bar{c}_{i}$ and $\eta_{i}$ do not have a pit at all. This is always the case for our model 1 where the single-phase $\mathrm{DO}_{3}$ region in the phase diagram is rather narrow (see figure 1(a)) and $c_{+}(c, T)$ is approximately equal to 0.5 everywhere in this region. On the other hand, near the $\eta$-APB both $\eta_{i}$ and $\zeta_{i}^{2}$ are strongly suppressed (see figure 3 ), and hence such boundaries should generate strong contrast in both the $\mathrm{B} 2$ and $\mathrm{DO}_{3}$ superstructure reflections. Both of these conclusions agree with the TEM observations of $\mathrm{Fe}-\mathrm{Al}$ alloys (see e.g. [5-7]).

\section{Kinetics of $\mathrm{A} 2 \rightarrow \mathrm{D0}_{3}$ transformation}

In this section we discuss the microstructural evolution after a rapid quench of a disordered A2 phase into a single-phase $\mathrm{DO}_{3}$ state. Some aspects of this evolution have been discussed by Allen and Cahn in their treatment of phase transformations in $\mathrm{Fe}-\mathrm{Al}$ alloys [1]. On the basis of thermodynamic considerations they suggested that the $\mathrm{A} 2 \rightarrow \mathrm{DO}_{3}$ transition should occur in two steps. First a transient B2-ordered state should arise via establishing of $\eta$-type concentration waves $\left(\boldsymbol{g}_{1}\right.$-waves) in relation (2). Then the $\mathrm{DO}_{3}$ order should appear via a development of $\zeta$-type concentration waves ( $\boldsymbol{g}_{2}$-waves). Allen and Cahn also mentioned that this suggestion seems to be supported by the observed microstructures which usually include many $\zeta$-APBs enclosed by much more extended $\eta$-APBs. However, such microstructures in the $\mathrm{Fe}-\mathrm{Al}$-type alloys can arise just due to an insufficiently rapid quench which can result in some annealing in the B2-ordered state passed during the quench; see figure 1(a).

Microscopical simulations can provide direct information about the kinetics of the $\mathrm{A} 2 \rightarrow \mathrm{DO}_{3}$ transition. We performed a 2D simulation of this transformation for our model 1 at $c=0.25, T^{\prime}=0.424$ (point $\alpha$ in figure 1(a)), and also simulated for comparison the $\mathrm{A} 2 \rightarrow \mathrm{B} 2$ transition at $c=0.35, T^{\prime}=0.7$ for the same model (point $\beta$ in figure $1(\mathrm{a})$ ). For 
both simulations we employed the same distribution of initial fluctuations $\delta c_{i}$ in the $192 \times 192$ simulation box, so the initial alloy states in these two simulations differ only in $c$ - and $T^{\prime}$ values. For model 2 we studied the $\mathrm{A} 2 \rightarrow \mathrm{DO}_{3}$ transformation with $2 \mathrm{D}$ simulations in the $128 \times 128$ simulation box at $c=0.133, T^{\prime}=0.35$ (point $\delta$ in figure $1(\mathrm{~b})$ ), and also with 3D simulations in the $40^{3} \times 2$ simulation box at $c=0.2, T^{\prime}=0.4$ (point $\varepsilon$ in figure 1(b)).

Some results of these simulations are presented in figures 4-7. Let us first discuss those for model 1 shown in figures 4 and 5. First of all, they confirm the above-mentioned suggestion of Allen and Cahn that the first stage of the $\mathrm{A} 2 \rightarrow \mathrm{DO}_{3}$ transformation is the transient $\mathrm{B} 2$ ordering: the microstructure for $t^{\prime}=3$ shown in figure 4 (a) corresponds to the B2-ordered state. This state is quite similar to that for the analogous A2 $\rightarrow$ B2 transition (figure 4(e)) - in particular, in the morphology of $\eta$-APBs (which depends mainly on the distribution of initial fluctuations
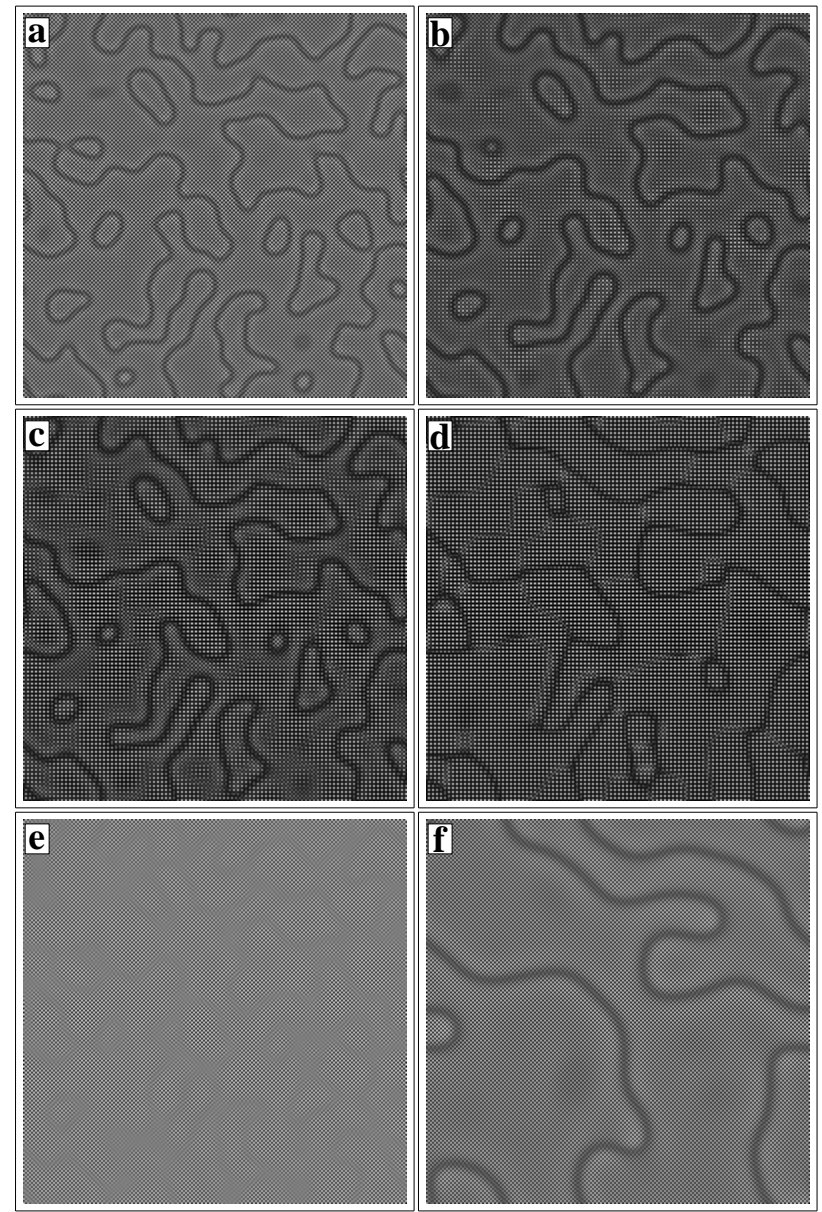

Figure 4. Frames (a)-(d): temporal evolution of an alloy model 1 under phase transformation $\mathrm{A} 2 \rightarrow \mathrm{D}_{3}$ at $c=0.25, T^{\prime}=0.424$ and the following values of reduced time $t^{\prime}$ : (a) 3 ; (b) 10 ; (c) 15; and (d) 50. The grey level varies linearly with $c_{i}$ between its minimum and maximum values from completely dark to completely bright. The simulation box contains $192 \times 192$ lattice sites. Frames (e) and (f): as frames (a)-(d), but for the A2 $\rightarrow$ B2 transformation at $c=0.35, T^{\prime}=0.7$ and the following values of $t^{\prime}$ : (e) 3 ; and (f) 50 . 

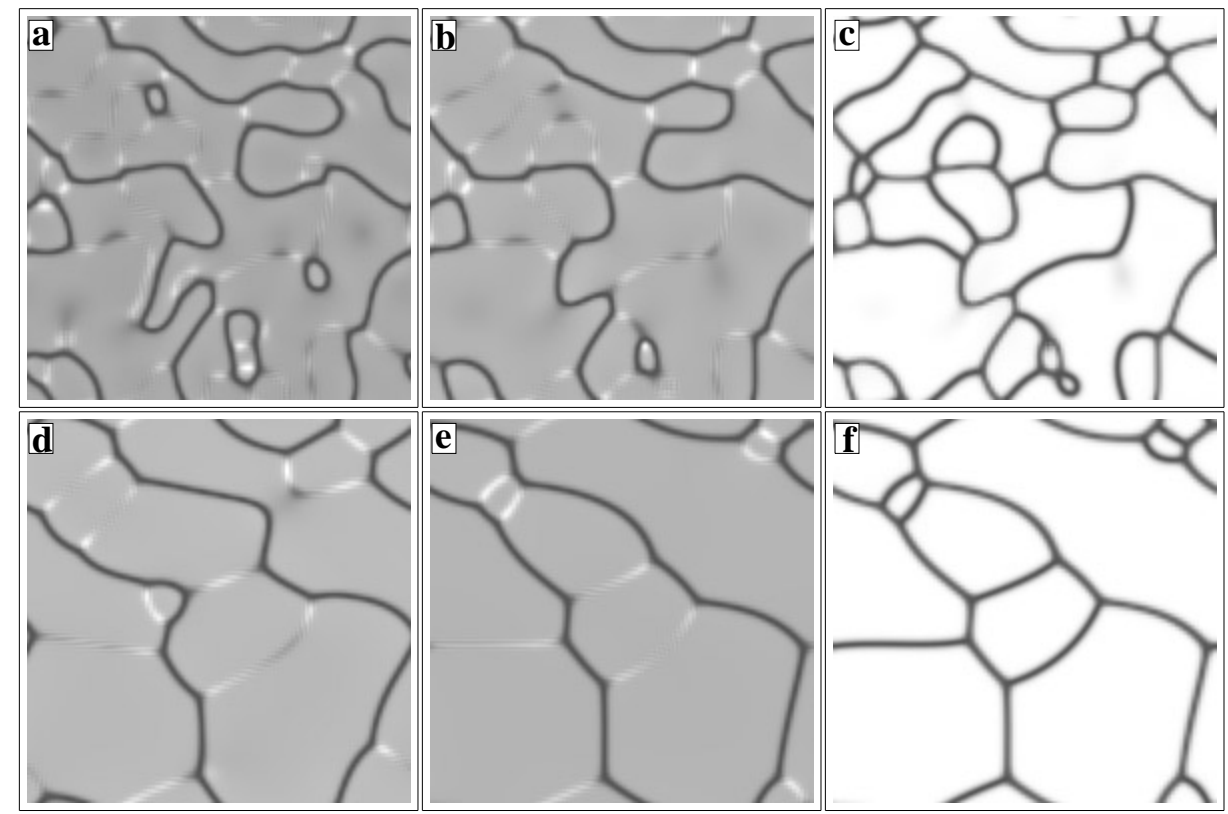

Figure 5. Temporal evolution of alloy model 1 under phase transformation $\mathrm{A} 2 \rightarrow \mathrm{D}_{3}$ at $c=0.25$, $T^{\prime}=0.424$ and the following values of reduced time $t^{\prime}$ : (a) 50; (b) and (c), 100; (d) 400; (e) and (f), 1000. Frames (a), (b), (d) and (e) are shown in $\eta^{2}$-representation, while frames (c) and (f) are shown in $\zeta^{2}$-representation. The grey level varies linearly with $\eta^{2}$ or $\zeta^{2}$ between its minimum and maximum values from dark to bright. State (a) is the same as in figure $4(d)$.

$\delta c_{i}$ being the same for both simulations). Minor differences between the microstructures in figures 4(a) and 4(e) merely show that at these early $t^{\prime}$ the microstructural evolution under the $\mathrm{A} 2 \rightarrow \mathrm{DO}_{3}$ transformation proceeds faster than that under the $\mathrm{A} 2 \rightarrow \mathrm{B} 2$ transformation. Figures 4(b) and 4(c) illustrate the subsequent creation and development of the $\mathrm{D}_{3}$-ordered regions within the initially ordered B2 domains. By $t^{\prime}=50$ (figure $4(\mathrm{~d})$ ) this process is mainly completed and the initial network of $\zeta$-APBs interlinking the initial $\eta$-APBs is well formed. The characteristic size of the $\mathrm{DO}_{3}$-ordered $\zeta$-APDs is the same as that of the initial B2-ordered $\eta$-APDs, but the shape of these $\zeta$-APDs is much more regular. The morphology of $\eta$-APBs at $t^{\prime}=50$ for the $\mathrm{A} 2 \rightarrow \mathrm{DO}_{3}$ transition (figures 4(d) and 5(a)) is still similar to that for the A2 $\rightarrow$ B2 transition (figure 4(f)), but it includes also a number of extra smaller ' $\eta$-APB loops' and ' $\eta$-APB segments'. Coarsening of these smaller structural units under the $\mathrm{A} 2 \rightarrow \mathrm{DO}_{3}$ transition is slowed down compared to the A2 $\rightarrow$ B2 transition since numerous $\zeta$-APBs tie these units with each other and thereby damp their motion.

Figures 5(a), 5(b), 5(d) and 5(e) shown in $\eta^{2}$-representation demonstrate good contrast from $\eta$-APBs and faint contrast from $\zeta$-APBs. On the other hand, figures 5(c) and 5(f) shown in $\zeta^{2}$-representation demonstrate good contrast from APBs of both types. The origin of this difference was discussed in section 4. It is also worth noting that different segments of $\zeta$-APBs in figure 5 show different contrast in the $\eta^{2}$-representation: some of them are brighter and some are darker than the bulk of the APDs. This effect is due to the fact that the $c, T$-point under consideration is very close to the $c_{+}(T)=0.5$ curve, and different regions of the sample can reside on different sides of this curve because of the fluctuations of local concentration. As $\eta_{i}$ has a pit to the left or a hump to the right of this curve, the $\zeta$-APBs in regions locally enriched or 

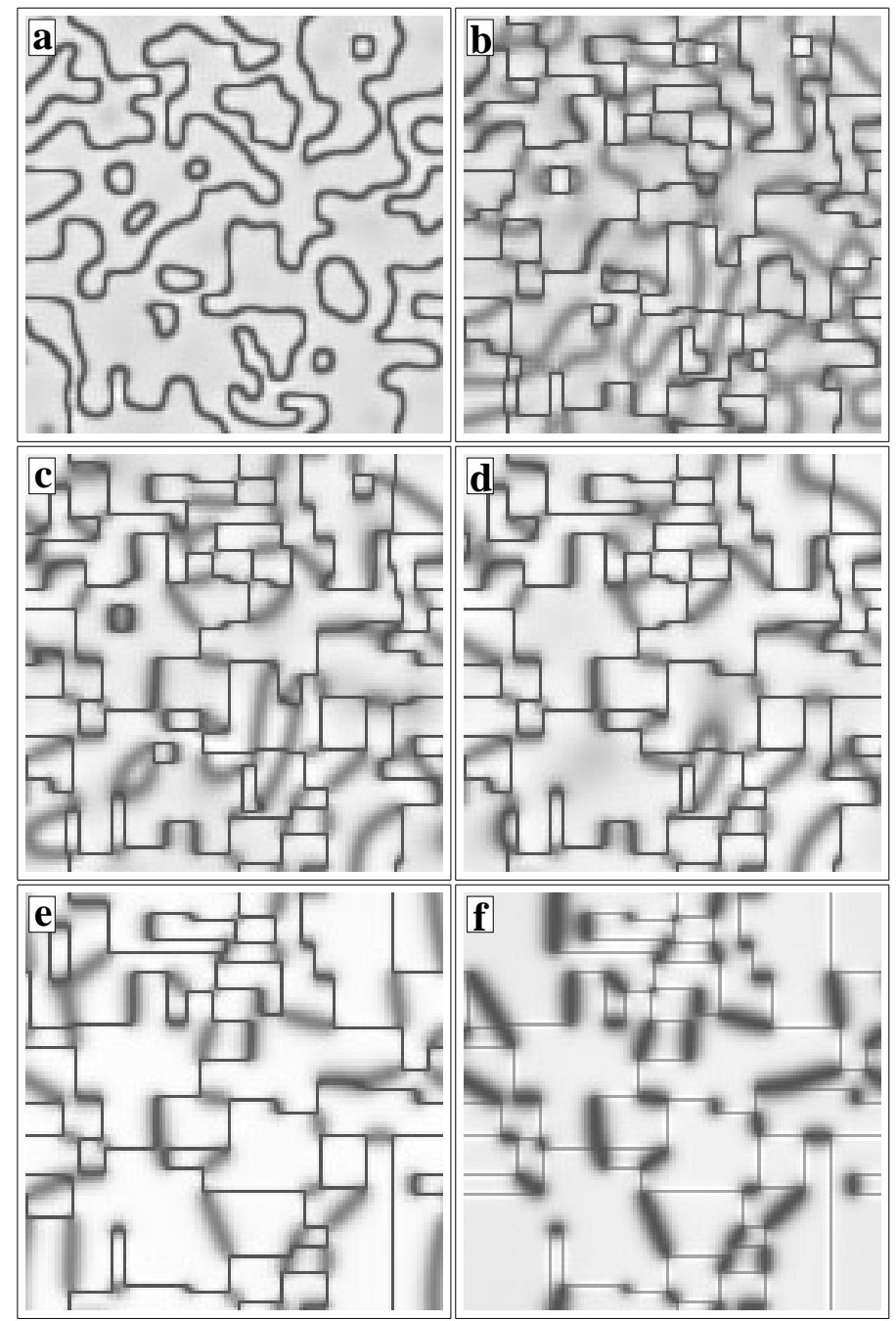

Figure 6. Temporal evolution of alloy model 2 under the phase transformation $\mathrm{A} 2 \rightarrow \mathrm{DO}_{3}$ at $c=0.133, T^{\prime}=0.35$ and the following values of reduced time $t^{\prime}$ : (a) 10 ; (b) 30 ; (c) 100 ; (d) 200 ; (e) 2000; and (f) 5000. Frames (a)-(e) are shown in $\eta^{2}$-representation, while frame (f) is shown in $\zeta^{2}$-representation. The simulation box contains $128 \times 128$ lattice sites. The shading is the same as in figure 5 .

depleted by the minority component show brighter or darker contrast in the $\eta^{2}$-representation. Such reverse contrast could possibly be observed in real Fe-Al-type alloys.

Another characteristic feature of the microstructures shown in figure 5 is the predominance of triple junctions of APBs. This predominance has a topological origin (the sum of displacement vectors of all of the APBs linked in a junction should be equal to zero) and is an inherent feature of all multivariant orderings with approximately isotropic free energy of the APBs. In particular, the microstructures observed under $\mathrm{L}_{2}$ ordering in $\mathrm{Cu}-\mathrm{Pd}$ alloys (figure 9 in [6]) are very similar to those shown in figure 5. Let us also note that the APDs formed under 


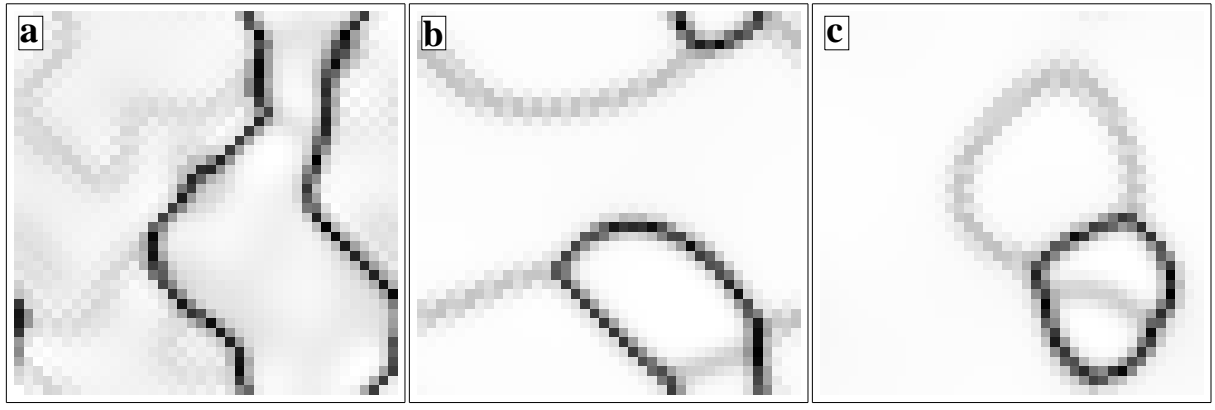

Figure 7. Temporal evolution of alloy model 2 in the 3D BCC simulation box of $40^{3} \times 2$ lattice sites under phase transformation $\mathrm{A} 2 \rightarrow \mathrm{DO}_{3}$ at $c=0.2, T^{\prime}=0.4$ shown in $\eta^{2}$-representation for the following values of reduced time $t^{\prime}$ and of the vertical coordinate $z$ : (a) $t^{\prime}=10, z=0$; (b) $t^{\prime}=100, z=0$; and (c) $t^{\prime}=100, z=20 a$. The grey level varies linearly with $\eta^{2}$ between its minimum and maximum values from dark to bright.

the multivariant $\mathrm{DO}_{3}$ ordering are mostly regularly shaped and approximately equiaxial — see e.g. figures 5(c) and 5(f) —unlike the elongated 'swirl-shaped' APDs characteristic of orderings with only two types of ordered domain-see e.g. figures 4(e), 4(f) and 10(a)-10(f), or figure 1 of [7].

Figure 6 shows the $\mathrm{A} 2 \rightarrow \mathrm{DO}_{3}$ transformation in our Fe-Si-type model 2 at $c=0.133$, $T^{\prime}=0.35$ (point $\delta$ in figure 1(b)). Figures 6(a)-6(e) are given in the $\eta^{2}$-representation and figure $6(\mathrm{f})$ in the $\zeta^{2}$-representation. The first stage of the transformation is again a transient B2 ordering, and the microstructure shown in figure 6(a) (similarly to that in figure 4(a)) represents this transient B2-ordered state. Absence of triple APB junctions indicates that the $\mathrm{D}_{3}$ ordering has not yet significantly developed over the sample.

In later stages of evolution, when the $\mathrm{DO}_{3}$ order parameter has virtually reached its equilibrium value (figures 6(b)-6(f)), a large proportion of sufficiently long $\eta$-APBs become very thin and aligned along the (10) directions parallel to their vector of displacement $\boldsymbol{b}_{d}=(1,0) a$ or $\boldsymbol{b}_{d}=(0,1) a$. In our 2D model 2 , such boundaries present an example of the so-called conservative APBs, which can be viewed as a half-space of the ordered domain shifted along the displacement vector parallel to the APB [34]. Without relaxation, the average concentration in each plane parallel to a conservative APB is the same as in the bulk of the ordered phase. The alignment of such boundaries in our 2D model 2, with the short-range second-neighbour interaction, is due to the fact that their interfacial energy is zero because they do not break the nearest- and next-nearest-neighbour bonds. Similar alignment is observed in a number of FCC alloys with $\mathrm{L}_{2}$ or $\mathrm{L}_{0}$ ordering-for example, in $\mathrm{Cu}_{3} \mathrm{Au}$ [6] where the conservative APBs oriented along the (100) planes do not alter the first-neighbour $\mathrm{Cu}-\mathrm{Au}$ bonds. In figure 6 one can also observe some tendency to formation of quadruple junctions of the conservative APBs (or rather two tightly bound triple junctions with a short segment of the interlinking $\zeta$-APB) and of the characteristic 'kinks' of such APBs. These effects are a natural consequence of the thermodynamical preference of the conservative APBs and are also observed experimentally in $\mathrm{Cu}_{3} \mathrm{Au}$ alloys (see figure 5 of [6]). Let us also note that figure 5(b) of [6], showing a HREM image of the quadruple APB junctions in the $\mathrm{Cu}_{3} \mathrm{Au}$ alloy, demonstrates that these quadruple junctions essentially consist of two triple APB junctions with a short interlinking segment of a non-conservative APB which is notably thicker than the aligned conservative APBs, just as in our figure 6. 
Among the generally isotropic network of $\eta$-APBs in figure 6(a) one can also notice several short and very thin segments strictly aligned along the (10) directions. These segments have been formed in the regions where the $\mathrm{D}_{3}$ ordering had already developed in the vicinity of the favourably oriented $\eta$-APBs.

Contrary to the case for the simulation shown in figure 5 , the point $c, T$ for the simulation shown in figure 6 (point $\delta$ in figure 1(b)) is far from the $c_{+}(T)=0.5$ curve. Hence the pits of $\eta_{i}$ at $\zeta$-APBs are here much more pronounced than that in model 1 shown in figure 2 . Therefore, both $\eta$-APBs and $\zeta$-APBs exhibit good contrast in the $\eta^{2}$-representation used in figures 6(a)-6(e).

It is interesting to note the peculiar coarsening kinetics in figure 6. Straight conservative boundaries with zero interfacial energy are virtually immobile unless they are driven by curved and diffuse non-conservative boundaries which freely 'leak' through the former. One can observe such a process, for example, at about $x=75 a, y=40 a$ and $x=100 a, y=75 a$ where the coordinates $x$ and $y$ are counted from the lower left corner of our simulation box of $128 \times 128$ sites.

Finally, in figure 7 we present some results of the 3D simulation for our model 2 at $c=0.2$, $T^{\prime}=0.4$ (point $\varepsilon$ in figure $1(\mathrm{~b})$ ). Unlike in the $2 \mathrm{D}$ case, there are no APBs in the $3 \mathrm{D} \mathrm{DO}_{3}$ system that do not alter the first- and second-neighbour bonds. Therefore, in the 3D version of our Fe-Si-type model 2 with two interaction constants, one should not expect to observe strictly aligned conservative APBs as in the 2D version of the same model. Indeed, figure 7 does not show such sharp alignment of APBs as one can see in figure 6. However, one can observe a notable tendency to alignment of both $\eta$ - and $\zeta$-APBs along the (110) and (100) planes. This means that these orientations have a lower interfacial free energy compared to other orientations (note, in particular, that for the nearest-neighbour interaction model the conservative $\zeta$-APBs in the $3 \mathrm{D}$ case would have zero interfacial energy). The preferential orientation of APBs along (110) and (100) directions shown in figure 7 is analogous to a similar microfaceting of APBs observed under the $\mathrm{L1}_{2}$ ordering in $\mathrm{Cu}_{3} \mathrm{Pd}$ alloy (figure 4 of [6]).

\section{Kinetics of $\mathrm{A} 2 \rightarrow \mathrm{A} 2+\mathrm{D0}_{3}$ transformation and effects of elastic interaction}

In their discussion of $\mathrm{A} 2 \rightarrow \mathrm{A} 2+\mathrm{B} 2$ and $\mathrm{A} 2 \rightarrow \mathrm{A} 2+\mathrm{DO}_{3}$ transformations in $\mathrm{Fe}-\mathrm{A} 1$ alloys, Allen and Cahn [1] noted that the first stage of these transitions (as well as that for the $\mathrm{A} 2 \rightarrow \mathrm{DO}_{3}$ transition) corresponds to a transient B2-ordered state, and as the A2 phase perfectly wets the $\eta$-APBs, it precipitates primarily on these APBs. Meanwhile, the wetting of the $\zeta$-APBs by the A2 phase appears to occur much more sluggishly, if at all (which can be related to a significant degree of B2 order within such APBs in the Fe-Al-type systems; see section 4). Similar observations were made by other authors [2,5]. Allen and Cahn also noted a 'surprising scarcity' of $\zeta$-APBs in their final $\mathrm{A} 2+\mathrm{D}_{3}$ microstructure (figure 9 of [1]). Effects of elastic interaction on microstructural evolution under the A2 $\rightarrow \mathrm{A} 2+\mathrm{B} 2$ transformation were discussed in a number of works by Khachaturyan and co-workers [8-10]. These authors used 2D models, an approximate kinetic equation (whose possible errors were discussed in [13]) and the above-mentioned asymptotic approximation of the elastic potential $v^{e l}$, while the results obtained were applied, in particular, to interpreting the microstructures observed under multivariant orderings in real alloys [11].

In this section we consider the $\mathrm{A} 2 \rightarrow \mathrm{A} 2+\mathrm{DO}_{3}$ transformation in our $\mathrm{Fe}-\mathrm{Al}$-type model 1 , paying special attention to the effect of multivariance of the ordering on microstructural evolution. We also discuss the elastic effects on this evolution, employing for the elastic interaction $v^{e l}$ a consistent model described in section 3 instead of its simplified version used in $[8-10]$. 
First, let us note that coalescence of ordered precipitates within the disordered matrix (which is an important coarsening mechanism at initial and intermediate stages of the phase transformation $[10,13]$ ) is possible only if these ordered domains are 'in phase', i.e. if they can be superposed by a lattice translation [10]. This is a rather stringent restriction in a multivariantly ordered alloy where the probability of the two randomly chosen APDs being in phase is equal to the inverse number of APD types, i.e. $1 / 4$ under $\mathrm{D}_{3}$ and $\mathrm{L}_{2}$ ordering or even $1 / 6$ under $\mathrm{L} 1_{0}$ ordering, while for $\mathrm{B} 2$ ordering it is $1 / 2$. Therefore, one can expect to observe far fewer interconnections between the in-phase APDs in the phase-separating alloy with a multivariant ordering compared to B2 ordering (as was also noted in [16]). Indeed, the evolution in the 2D models of Khachaturyan and co-workers [10,11] included many coalescence events even in alloys with a small volume fraction of the ordered phase considered in these works. Meanwhile, the experimental microstructures, for example in the two-phase $\gamma-\gamma^{\prime}$ superalloys with $\mathrm{L}_{2}$ ordering, show quasi-regular arrays of non-coalescing adjacent APDs even in alloys with a very high volume fraction of the ordered phase (see e.g. [39]), which can hardly arise in alloys with only two types of ordered domain.

To illustrate these considerations and to study the effect of both multivariance and elastic interaction on microstructural evolution, we simulated the $\mathrm{A} 2 \rightarrow \mathrm{A} 2+\mathrm{D}_{3}$ phase transition in our Fe-Al-type model 1 at $c=0.187, T^{\prime}=0.424$, both without elastic interaction when the parameter $\lambda$ in (30) is zero (figure 8), and for the significant, but realistic value, $\lambda=0.3$ (figure 9). For comparison we also simulated the $\mathrm{A} 2 \rightarrow \mathrm{A} 2+\mathrm{B} 2$ phase transition at $c=0.325, T^{\prime}=0.424$, again at $\lambda=0$ and $\lambda=0.3$ (figures 10 and 11), for a similar model used in [13] which at $\lambda=0$ corresponds to the interactions $v_{1}>0, v_{2} / v_{1}=-0.8$, $v_{3} / v_{1}=-0.5$ and $v_{n} \geqslant 4=0$. The $c, T$-points on the phase diagrams were chosen so as to make the equilibrium volume fraction of the ordered phase identical for all models. For all four models the critical B2-ordering temperature was taken as $T_{c}=1000 \mathrm{~K}$. Elastic interactions in (19), (28), (29) are determined by one energetic parameter (e.g. $c_{11} \Omega$ ) and by two ratios of elastic constants (e.g. $c_{12} / c_{11}$ and $c_{44} / c_{11}$ ); for these parameters we took the experimental values for BCC iron, where the $c_{i k}$ (in Mbar) are $c_{11} \simeq 2.335, c_{12} \simeq 1.355$ and $c_{44} \simeq 1.18$, while $\Omega$ is $11.78 \AA^{3}$. Then equation (30) with $\lambda=0.3$ determines the concentrational expansion coefficient $u_{0}$ in (28) or (29) which in the $2 \mathrm{D}$ case gives $u_{0} \simeq 0.115$ and in the $3 \mathrm{D}$ case gives $u_{0} \simeq 0.084$, both values being quite reasonable and typical for real alloys.

Considering both $\mathrm{D}_{3}$ and $\mathrm{B} 2$ ordering with elastic interaction we adjusted the chemical interaction constants $v_{n}^{c}$ for the model with $\lambda=0.3$ such that its phase diagram would coincide with that for the model with $\lambda=0$. As mentioned in section 3, the MFA phase diagram including the $\mathrm{A} 2, \mathrm{~B} 2, \mathrm{D}_{3}$ and $\mathrm{A} 2+\mathrm{B} 2$ or $\mathrm{A} 2+\mathrm{D}_{3}$ regions is determined by the full interaction potential $v(\boldsymbol{k})=v^{c}(\boldsymbol{k})+v^{e l}(\boldsymbol{k})$ at three values of $\boldsymbol{k}: 0, \boldsymbol{g}_{1}$ and $\boldsymbol{g}_{2}$ (or, in the 2D case, $0, g_{1}$ and $g_{2 x}$ or $\boldsymbol{g}_{2 y}$ ), while $v^{e l}(0)$ corresponds to the asymptotic expression (29) (or (28) in the 2D case) for the elastically soft direction $\boldsymbol{n}=[1,0,0]$ (or $\boldsymbol{n}=[1,0]$ ) $[8,9,36]$. Hence for the $\mathrm{D}_{3}$-ordering model such adjustment (performed for the given $v^{e l}$ determined by equations (19)-(27) with the above-mentioned values of $c_{i k}$ and $\Omega$ ) provides three equations for the chemical interactions $v_{1}^{c}, v_{2}^{c}$ and $v_{3}^{c}$. The solutions $v_{n}^{c}$ of these equations correspond to the model with $\lambda=0.3$, while equations (7) correspond to the model with $\lambda=0$. To treat all of our four models as similarly as possible, the same procedure was also used for the B2 ordering: we again supposed the values of $v(0), v\left(\boldsymbol{g}_{1}\right)$ and $v\left(\boldsymbol{g}_{2 x}\right)$ to be the same for the models with $\lambda=0.3$ and $\lambda=0$, even though the value of $v\left(g_{2 x}\right)$ here is not determined by the MFA phase diagram.

Let us first discuss the case of small elastic anisotropy, $\lambda=0$ (which corresponds, in particular, to real $\mathrm{Fe}-\mathrm{Al}$ alloys [1]). The evolution under the $\mathrm{A} 2 \rightarrow \mathrm{A} 2+\mathrm{D}_{3}$ transition is shown in figure 8 , and that under the $\mathrm{A} 2 \rightarrow \mathrm{A} 2+\mathrm{B} 2$ transition is shown in figure 10 


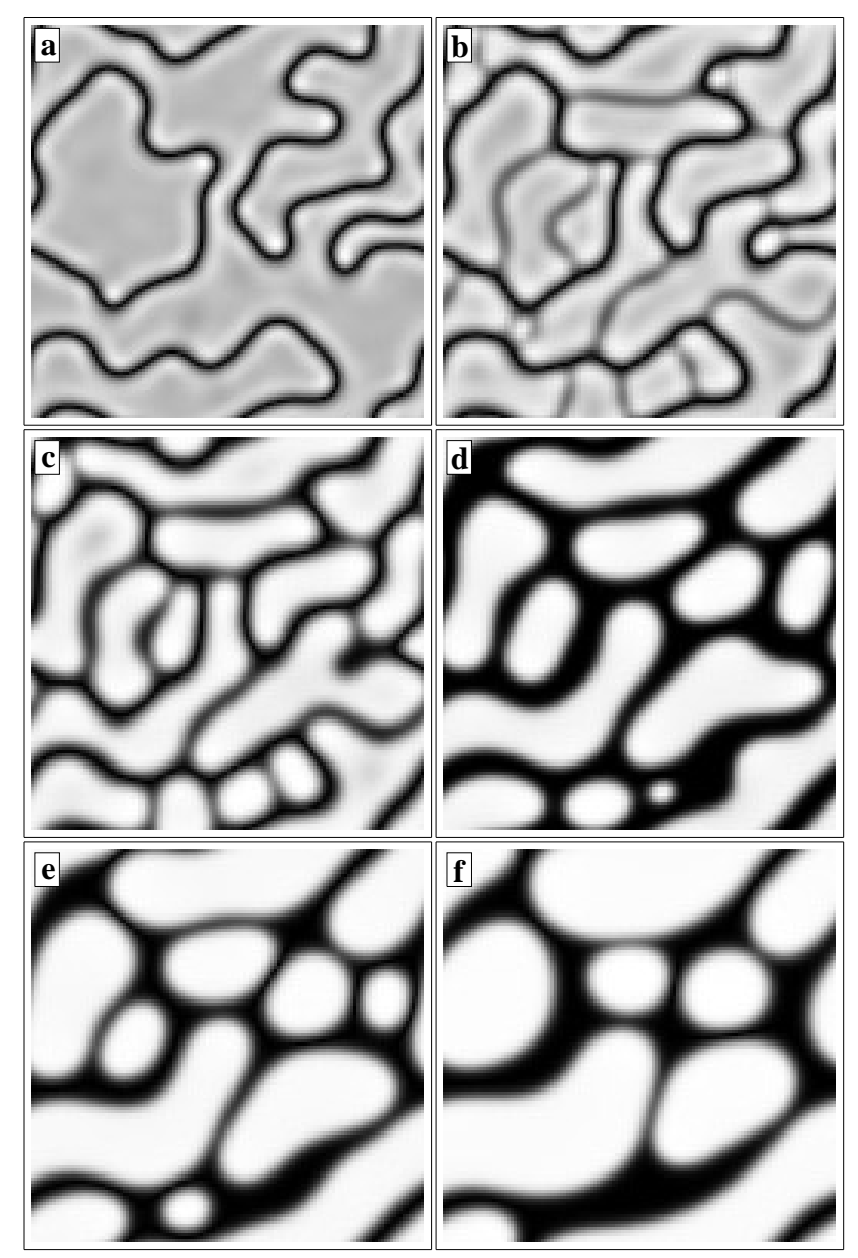

Figure 8. Temporal evolution of alloy model 1 with $\lambda=0$ under $\mathrm{A} 2 \rightarrow \mathrm{A} 2+\mathrm{D}_{3}$ transformation at $c=0.187, T^{\prime}=0.424$ shown in $\eta^{2}$-representation for the following values of reduced time $t^{\prime}$ : (a) 10; (b) 30; (c) 100; (d) 500; (e) 1000; and (f) 2000. The simulation box contains $128 \times 128$ lattice sites. The shading is the same as in figure 4 .

(see also figure 5 in reference [13] which differs from figure 10 only due to the difference in initial fluctuations $\delta c_{i}$ ). The first stages of the two transformations (figures 8(a) and 10(a)) correspond to a transient B2-ordered state and are quite similar to each other, just like those in figures 4(a) and 4(e) for the $\mathrm{A} 2 \rightarrow \mathrm{DO}_{3}$ and $\mathrm{A} 2 \rightarrow \mathrm{B} 2$ transitions. In the further course of the $\mathrm{A} 2 \rightarrow \mathrm{A} 2+\mathrm{DO}_{3}$ transformation the initial $\mathrm{B} 2$-ordered domains split into smaller $\zeta$ APDs separated by $\zeta$-APBs (figure 8(b)), and the microstructures become very different from those observed under the $\mathrm{A} 2 \rightarrow \mathrm{A} 2+\mathrm{B} 2$ transition. The disordered A2 phase begins to wet the $\eta$-APBs, and also the $\zeta$-APBs, but this latter process is very sluggish (in agreement with experimental observations $[1,2,5])$ and the main mechanism of coarsening appears to be the dissolution of smallest 'out-of-phase' $\zeta$-APDs; see figures 8(c)-8(f). The outer edges of $\zeta$ APBs are wetted by the A2 phase, and the remaining segments of these $\zeta$-APBs between the adjacent $\zeta$-ordered precipitates get rather short. The main features of our final microstructure 


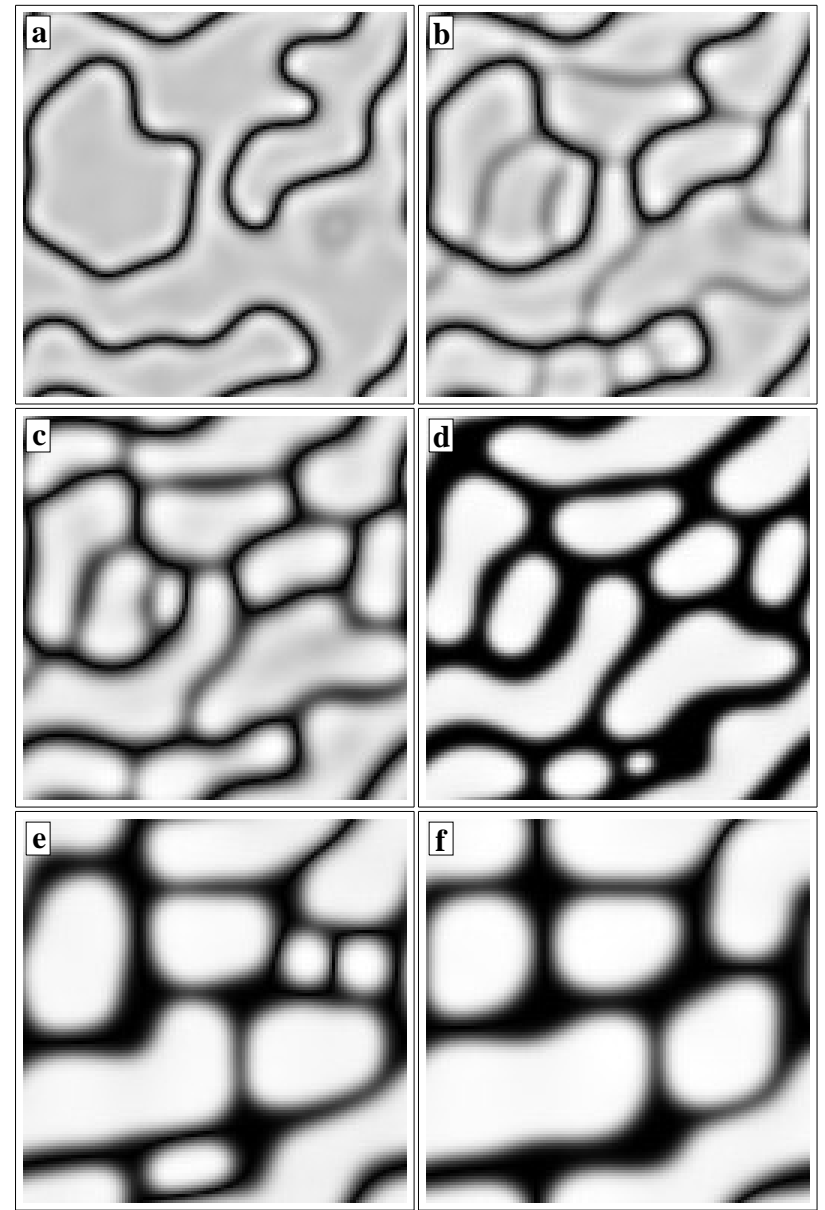

Figure 9. As figure 8, but for $\lambda=0.3$.

in figure 8(f) seem to be very similar to those observed in figure 9 of [1], including the absence of extended $\zeta$-APBs noted by Allen and Cahn. Comparison of figures 8(f) and 10(f) also illustrates a drastic effect of multivariance on the microstructure: the ordered precipitates in figure 8(f) are much more compact and regularly shaped than those in figure 10(f).

Figures 9 and 11 correspond to the models with $\lambda=0.3$ and illustrate the effect of elastic anisotropy on microstructural evolution. In the first stages of phase transformations, when no phase separation has yet occurred, this effect is insignificant (compare figures 9(a), 9(b) with $8(a), 8(b)$ or $11(a), 11(b)$ with $10(a), 10(b))$, but at later stages, when phase separation starts, this effect becomes quite pronounced. Under the $\mathrm{A} 2 \rightarrow \mathrm{A} 2+\mathrm{D}_{3}$ transition both $\zeta$-APBs and the $\mathrm{A} 2-\mathrm{D}_{3}$ interphase boundaries tend to align along the elastically soft (10) directions, and the final panel, figure 9(f), shows an array of approximately rectangular $\mathrm{D}_{3}$ ordered precipitates, instead of the rounded 'droplets' shown in figure 8(f). The microstructure in figure 9(f) is similar to those observed under the $\mathrm{A} 2 \rightarrow \mathrm{A} 2+\mathrm{D}_{3}$ transition in $\mathrm{Fe}-\mathrm{Ga}$ alloys [4], and it also resembles the quasi-regular arrays of $\mathrm{L}_{2}$-ordered precipitates observed in $\gamma-\gamma^{\prime}$ alloys [39]. Comparison of figures 9 and 11 again demonstrates a great difference in 


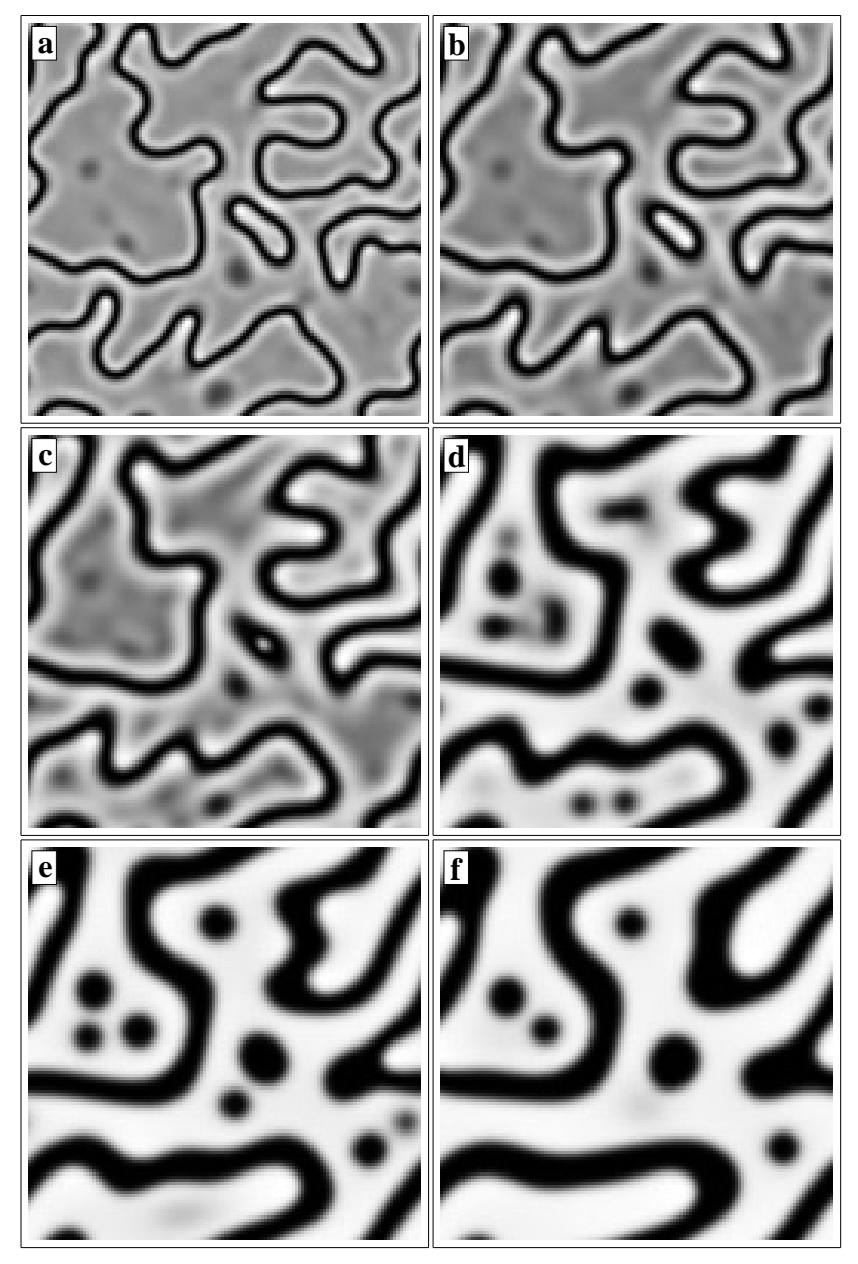

Figure 10. Temporal evolution of an alloy model from [13] with $\lambda=0$ under $\mathrm{A} 2 \rightarrow \mathrm{A} 2+\mathrm{B} 2$ transformation at $c=0.325, T^{\prime}=0.424$ shown in $\eta^{2}$-representation for the following values of reduced time $t^{\prime}$ : (a) 10; (b) 30; (c) 100; (d) 500; (e) 1000; and (f) 2000. The simulation box contains $128 \times 128$ lattice sites. The shading is the same as in figure 4 .

microstructure for the multivariant and two-variant ordering. For the $\mathrm{A} 2 \rightarrow \mathrm{A} 2+\mathrm{B} 2$ transition the microstructures correspond to the formation of interconnected ordered and disordered rods (figure 11), instead of the approximately equiaxial ordered precipitates embedded in the disordered phase for the $\mathrm{A} 2 \rightarrow \mathrm{A} 2+\mathrm{DO}_{3}$ transition (figure 9). The microstructures in figure 11 are similar to those observed by Oki et al [3] under the $\mathrm{B} 2 \rightarrow \mathrm{B} 2+\mathrm{DO}_{3}$ transition in the $\mathrm{Fe}-\mathrm{Si}$ alloy. This similarity is quite natural since, as mentioned in section 4 , the $\mathrm{B} 2 \rightarrow \mathrm{B} 2+\mathrm{DO}_{3}$ transition from a single-domain $\mathrm{B} 2$ state is topologically equivalent to the $\mathrm{A} 2 \rightarrow \mathrm{A} 2+\mathrm{B} 2$ transition.

The microstructures in figures 11(a)-11(f) are qualitatively similar to those obtained by Khachaturyan and co-workers $[8,9]$ for the $\mathrm{A} 2 \rightarrow \mathrm{A} 2+\mathrm{B} 2$ transition in a similar model at similar values of concentration and temperature. However, the characteristic size of the microstructures (e.g. the width of the precipitates) in [8,9] is about 3-4 times smaller than that 

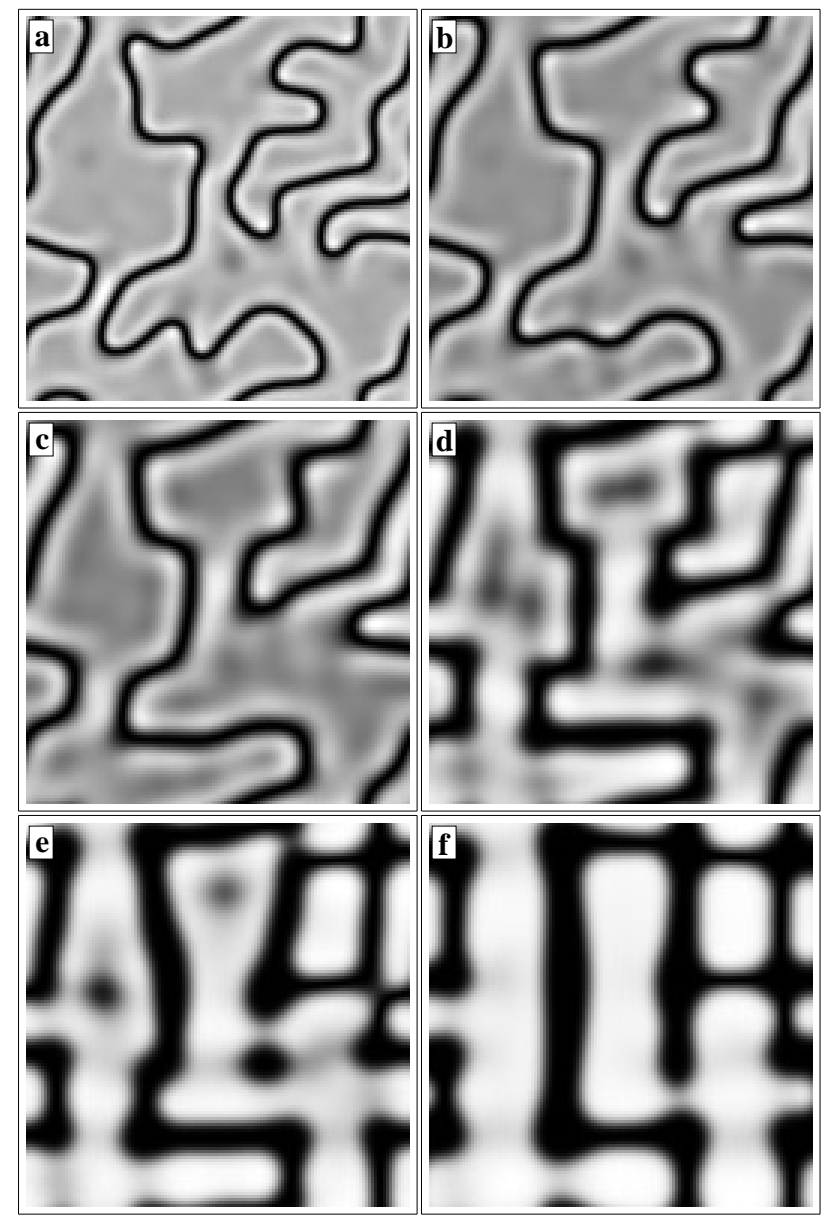

Figure 11. As figure 10, but for $\lambda=0.3$.

in our figure 11. Khachaturyan and co-workers $[8,9]$ used a slightly larger value of the elastic anisotropy parameter $\lambda=0.53$ compared to our $\lambda=0.3$, but this can hardly explain such notable difference. Most probably, it arises mainly because Khachaturyan and co-workers employed the asymptotic form of the elastic potential $v^{e l}$ mentioned in section 3 instead of the full expression (19). Our studies showed that such an approximation results in a notable exaggeration of the elastic anisotropy effects, which can lead to the above-mentioned distortion of microstructures.

\section{Kinetics of $\mathrm{A2} \rightarrow \mathrm{B} 2+\mathrm{D0}_{3}$ and $\mathrm{D0}_{3} \rightarrow \mathrm{B} 2+\mathrm{D0}_{3}$ transformations in Fe-Si-type alloys}

In this section we discuss the kinetic behaviour and evolution of conservative and nonconservative $\mathrm{APBs}$ under phase transformations involving both $\mathrm{D}_{3}$ and $\mathrm{B} 2$ ordering. To this end we describe the simulations of the $\mathrm{A} 2 \rightarrow \mathrm{B} 2+\mathrm{D}_{3}$ and $\mathrm{D}_{3} \rightarrow \mathrm{B} 2+\mathrm{D}_{3}$ phase transitions in our Fe-Si-type model 2.

Figure 12 illustrates the $\mathrm{A} 2 \rightarrow \mathrm{B} 2+\mathrm{D}_{3}$ transformation after a rapid quench of the 


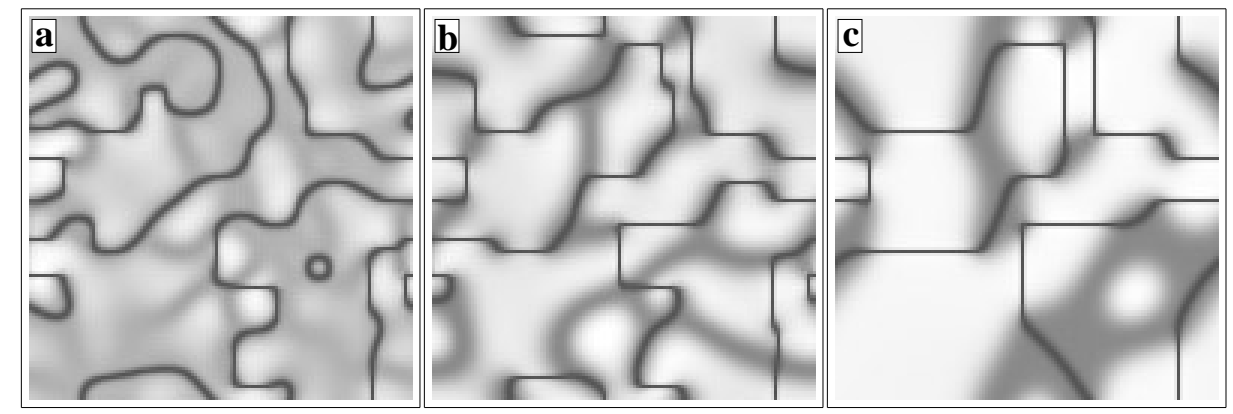

Figure 12. Temporal evolution of alloy model 2 under $\mathrm{A} 2 \rightarrow \mathrm{B} 2+\mathrm{D}_{3}$ transformation at $c=0.14$, $T^{\prime}=0.4$ shown in $\eta^{2}$-representation for the following values of reduced time $t^{\prime}$ : (a) 50; (b) 200; and (c) 2000. The shading is the same as in figure 7 . The simulation box contains $128 \times 128$ lattice sites.

disordered alloy with $c=0.14$ to the temperature $T^{\prime}=0.4$ which corresponds to the point $v$ in figure 1(b) with approximately $30 \%$ volume fraction of the B2 phase in equilibrium. In figure 12 we use the $\eta^{2}$-representation; the light areas correspond to the $\mathrm{D}_{3}$-ordered regions, the grey areas to the B2-ordered regions or to $\zeta$-APBs and the dark lines to $\eta$-APBs.

The first stages of the transformation shown in figures 12(a) and 12(b) are similar to those for the $\mathrm{A} 2 \rightarrow \mathrm{DO}_{3}$ transition in the same model (figures 6(a) and 6(b)). The larger scale of the initially formed $\mathrm{B} 2$ and then $\mathrm{DO}_{3}$ domains in figure 12 compared to figure 6 is due to the fact that point $v$ in figure 1 (b) is much closer to $\mathrm{B} 2$ - and $\mathrm{D}_{3}$-ordering spinodals than point $\delta$ corresponding to the alloy states shown in figure 6 . In figures 12(a), 12(b) one can observe the formation of strictly aligned conservative $\eta$-APBs, just as in figure 6 . Note that at $t^{\prime}=200$ (figure 12(b)) there is still no phase separation, and the microstructure includes only $\mathrm{D}_{3}$-ordered APDs separated by $\eta$ - or $\zeta$-APBs. After this initial stage of an approximately congruent ordering, the phase separation $\mathrm{DO}_{3} \rightarrow \mathrm{B} 2+\mathrm{DO}_{3}$ starts with the precipitation of the B2 phase on $\zeta$-APBs; see figure 12(c). At the same time, the conservative $\eta$-APBs (or their aligned 'conservative segments') are not wetted by the B2 phase and remain immobile and aligned. This difference is due to the fact that $\zeta$-APBs, unlike $\eta$-APBs, are locally B2 ordered (see section 4), and hence they are natural embryos for the formation of the B2 phase (just as $\eta$-APBs are natural embryos for the formation of the A2 phase). The above-described 'reappearance' of the $\mathrm{B} 2$ phase at non-conservative $\zeta$-APBs after the initial congruent $\mathrm{D}_{3}$ ordering was experimentally observed under the $\mathrm{B} 2 \rightarrow \mathrm{B} 2+\mathrm{DO}_{3}$ phase transition in $\mathrm{Fe}-\mathrm{Si}$ alloys [4].

Figure 13 illustrates the $\mathrm{DO}_{3} \rightarrow \mathrm{B} 2+\mathrm{DO}_{3}$ phase transition after a rapid heating of a single-phase $\mathrm{D}_{3}$ alloy from point $\delta$ to point $\sigma$ in figure $1(\mathrm{~b})$. For the initial $\mathrm{D}_{3}$ state we took the final state of the $\mathrm{A} 2 \rightarrow \mathrm{DO}_{3}$ transformation described in section 5 and shown in figure 6(f). Figures 13(a) and 13(b) (as well as figure 6(f)) are given in the $\zeta^{2}$-representation, and figures 13(c) and 13(d) are given in the $\eta^{2}$-representation, while figures 13(b) and 13(c) show the same alloy state in these two different representations. The lighter areas in figures 13(a) and 13(b) correspond to $\mathrm{D}_{3}$-ordered regions and the darker areas to $\mathrm{B} 2$-ordered regions or to $\zeta$ - or $\eta$-APBs, while the shading in figures 13(c) and 13(d) is the same as in figure 12.

Figures 13(a)-13(c) show that the B2 phase starts to precipitate at non-conservative $\zeta$ $\mathrm{APBs}$ (just as for the $\mathrm{A} 2 \rightarrow \mathrm{B} 2+\mathrm{DO}_{3}$ transition in figure 12), in agreement with the observations of Matsumura et al [4] for the $\mathrm{DO}_{3} \rightarrow \mathrm{B} 2+\mathrm{DO}_{3}$ transition in Fe-Si alloys. Meanwhile, the 


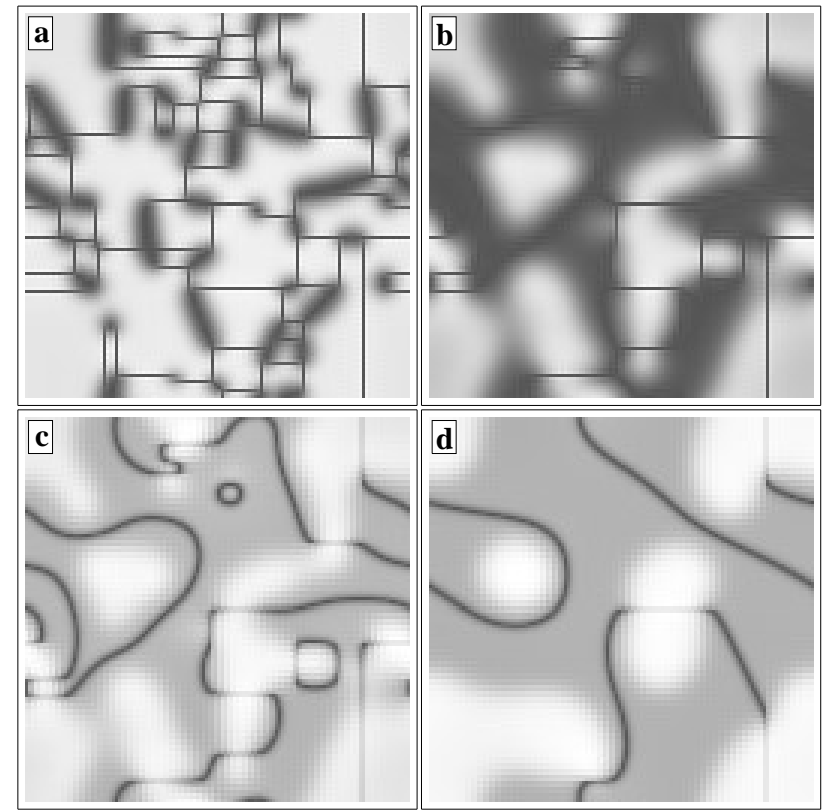

Figure 13. Temporal evolution of alloy model 2 under $\mathrm{D}_{3} \rightarrow \mathrm{B} 2+\mathrm{D}_{3}$ transformation after the state shown in figure 6(f) is rapidly heated to $T^{\prime}=0.4$ for the following values of reduced time $t^{\prime}$ : (a) 5; (b) and (c), 100; (d) 1000. Frames (a) and (b) are shown in $\zeta^{2}$-representation, while frames (c) and (d) are shown in $\eta^{2}$-representation. The shading is the same as in figure 5. The simulation box contains $128 \times 128$ lattice sites.

conservative $\eta$-APBs are virtually unaffected by the phase transformation unless the $\zeta$-APDs adjacent to these APBs are transformed into the B2 phase. Figures 13(c), 13(d) also show that within the B2 phase the $\eta$-APBs become thick and non-conservative, while within the $\mathrm{DO}_{3}$-ordered regions they remain thin, aligned and immobile.

\section{Conclusions}

Let us summarize the main results of this work. We used the earlier-described master equation approach and the kinetic mean-field approximation [17-20] to study the microstructural features of phase transformations with multivariant orderings. To this end we considered a number of phase transitions involving $\mathrm{DO}_{3}$-type orderings using $2 \mathrm{D}$ and $3 \mathrm{D}$ simulations for two alloy models which qualitatively correspond to $\mathrm{Fe}-\mathrm{Al}$ - and $\mathrm{Fe}-\mathrm{Si}$-type alloys with relatively long-range and short-range interactions, respectively. We also suggested a model for describing the effect of elastic anisotropy on microstructural evolution more consistently than previously, and employed it to study this effect under phase transformations of phase separation with ordering.

We studied the microscopical structure of different antiphase boundaries (APBs) in the $\mathrm{DO}_{3}$ phase, $\zeta$-APBs and $\eta$-APBs, which correspond to the vanishing within the APB of the $\mathrm{D}_{3}$ order parameter $\zeta$ or the $\mathrm{B} 2$ order parameter $\eta$, respectively. We have shown that at a $\zeta$-APB both the local B2 order parameter $\eta_{i}$ and the local mean concentration $\bar{c}_{i}$ have either a pit (when the $c, T$-point is to the left of the curve $c+\eta_{0}=0.5$ in the $c, T$-plane where $\eta_{0}$ is the equilibrium value of $\eta$ at the given concentration $c$ and temperature $T$ ), or a hump (when 
the $c, T$-point is to the right of the curve $c+\eta_{0}=0.5$ ), while for the $c, T$-values near the curve $c+\eta_{0}=0.5$ this pit or hump is small. Experimental observations showing faint [111] contrast on $\zeta$-APBs in the $\mathrm{D0}_{3}$-ordered $\mathrm{Fe}_{3} \mathrm{Al}$-type alloys [5-7] support this conclusion.

Next we describe the results of our simulations for the following transformations:

(a) $\mathrm{A} 2 \rightarrow \mathrm{DO}_{3}$ transition in both $\mathrm{Fe}-\mathrm{Al}$ - and Fe-Si-type alloys; see figures 4-7;

(b) $\mathrm{A} 2 \rightarrow \mathrm{A} 2+\mathrm{DO}_{3}$ transition in a Fe-Al-type alloy compared to the $\mathrm{A} 2 \rightarrow \mathrm{A} 2+\mathrm{B} 2$ transition in a similar model studied in [13]; see figures 8-11; and

(c) $\mathrm{A} 2 \rightarrow \mathrm{B} 2+\mathrm{DO}_{3}$ and $\mathrm{DO}_{3} \rightarrow \mathrm{B} 2+\mathrm{DO}_{3}$ transitions in a Fe-Si-type alloy; see figures 12 and 13 .

We observed a number of peculiar microstructural features connected with the multivariance of the ordering, in particular:

(i) Ordering of the initially disordered alloy quenched to any $c, T$-point below the B2-ordering spinodal, whether it be in the single-phase $\mathrm{DO}_{3}$, two-phase $\mathrm{A} 2+\mathrm{DO}_{3}$, or two-phase $\mathrm{B} 2+\mathrm{DO}_{3}$ region, develops through a transient $\mathrm{B} 2$ ordering, in accordance with the considerations of Allen and Cahn [1]; see figures 4, 6, 8 and 9.

(ii) The network of APBs in a single-phase multivariantly ordered alloy contains a lot of triple junctions (or triplanar lines in 3D systems) which makes the APDs much more regularly shaped and equiaxial compared to the 'swirl-shaped' APDs arising under orderings with only two types of ordered domain; see figures 4-7 and 10(a).

(iii) Due to the above-mentioned topological features of multivariant orderings, the ordered precipitates formed under the $\mathrm{A} 2 \rightarrow \mathrm{A} 2+\mathrm{D}_{3}$ phase separation are much more regularly shaped than the 'swirl-shaped' precipitates formed under the A2 $\rightarrow$ A2 + B2 transition. This difference is accentuated when significant elastic anisotropy is present which results in the formation of approximately rectangular ordered domains within the disordered matrix under the $\mathrm{A} 2 \rightarrow \mathrm{A} 2+\mathrm{DO}_{3}$ transition compared to the network of elongated rodlike (or plate-like, in 3D systems) precipitates for the $\mathrm{A} 2 \rightarrow \mathrm{A} 2+\mathrm{B} 2$ transition; see figures 8-11.

(iv) Under the $\mathrm{A} 2 \rightarrow \mathrm{A} 2+\mathrm{DO}_{3}$ phase transition the disordered $\mathrm{A} 2$ phase wets the $\zeta$-APBs, but this process is notably more sluggish compared to the wetting of $\eta$-APBs by the A2 phase. However, at later stages of the $\mathrm{A} 2 \rightarrow \mathrm{A} 2+\mathrm{DO}_{3}$ transition and in the absence of significant elastic effects, the $\zeta$-APBs are mostly wetted by the A2 phase, while the remaining segments of these APBs become short, and the microstructure includes few or no extended $\zeta$-APBs.

(v) In our Fe-Si-type model 2 there is a tendency for the formation of strictly aligned, 'conservative' $\eta$-APBs with low interfacial energy due to their special topology; see figures $6,12,13$. These APBs are very stable under all types of phase transformation and much thinner than the 'non-conservative' APBs.

(vi) Preference of the conservative APBs favours the formation of the bound pairs of triple APB junctions which look like quadruple APB junctions at low resolution.

(vii) Under the $\mathrm{DO}_{3} \rightarrow \mathrm{B} 2+\mathrm{DO}_{3}$ transition the $\mathrm{B} 2$ phase wets the non-conservative $\zeta$-APBs, while the conservative $\eta$-APBs are not wetted; see figure 13.

As was repeatedly noted in sections 5-7, many results of our simulations, including those mentioned in points (i)-(vii), agree well with the experimental observations of multivariant orderings described in references $[1-7,34,39]$. This agreement can illustrate the opportunities to use the master equation approach of references [17-20] for the microscopical studies of most of the various problems of microstructural evolution in alloys. 


\section{Acknowledgments}

The authors are much indebted to V Yu Dobretsov for his great help, as well as to Georges Martin, for numerous stimulating discussions. This work was supported by the Russian Fund of Basic Research, Grant No 97-02-17842.

\section{References}

[1] Allen S M and Cahn J W 1976 Acta Metall. 24425

[2] Allen S M 1977 Phil. Mag. 36181

[3] Oki K, Matsumura S and Eguchi T 1987 Phase Transitions 10257

[4] Matsumura S, Oyama H and Oki K 1989 Mater. Trans. JIM 30695

[5] Park J W and Moon I G 1992 Mater. Sci. Eng. A 152341

[6] Loiseau A, Ricolleau C, Potez L and Ducastelle F 1994 Solid-Solid Phase Transformations ed W C Johnson et al (Warrendale, PA: Minerals, Metals and Materials Society) p 385

[7] Korner A 1995 Phil. Mag. Lett. 7221

[8] Chen L-Q, Wang Y Z and Khachaturyan A G 1991 Phil. Mag. Lett. 64241

[9] Chen L-Q, Wang Y Z and Khachaturyan A G 1994 Statics and Dynamics of Alloy Phase Transformations (NATO Advanced Study Institute, Series B: Physics, vol 319) ed A Gonis and P E A Turchi (New York: Plenum) p 587

[10] Wang Y and Khachaturyan A G 1994 Scr. Metall. Mater. 311425

[11] Wang Y, Chen L-Q and Khachaturyan A G 1994 Solid-Solid Phase Transformations ed W C Johnson et al (Warrendale, PA: Minerals, Metals and Materials Society) p 245

[12] Dobretsov V Yu, Martin G, Soisson F and Vaks V G 1995 Europhys. Lett. 31417

[13] Dobretsov V Yu, Vaks V G and Martin G 1996 Phys. Rev. B 543227

[14] Gorentsveig V I, Fratzl P and Lebowitz J L 1997 Phys. Rev. B 552912

[15] Frontera C, Vives E, Castan T and Planes A 1997 Phys. Rev. B 55212

[16] Wang Y, Banerjee D, Su C C and Khachaturyan A G 1998 Acta Mater. 462983

[17] Gouyet J-F 1993 Europhys. Lett. 21335

[18] Vaks V G, Beiden S V and Dobretsov V Yu 1995 Pis. Zh. Eksp. Teor. Fiz. 6165 (Engl. Transl. 1995 JETP Lett. 61 68)

[19] Vaks V G 1996 Pis. Zh. Eksp. Teor. Fiz. 63447 (Engl. Transl. 1996 JETP Lett. 63 471)

[20] Belashchenko K D and Vaks V G 1998 J. Phys.: Condens. Matter 101965

[21] Belashchenko K D and Vaks V G 1997 Zh. Eksp. Teor. Fiz. 112714 (Engl. Transl. 1997 Sov. Phys.-JETP 85 390)

[22] Nastar M, Martin G and Dobretsov V Yu 1999 Phil. Mag. A at press

[23] Contreras-Solorio D A, Mejia-Lira F, Moran-Lopez J L and Sanchez J M 1988 Phys. Rev. B 38481

[24] Binder K 1994 Statics and Dynamics of Alloy Phase Transformations (NATO Advanced Study Institute, Series B: Physics, vol 319) ed A Gonis and P E A Turchi (New York: Plenum) p 467

[25] Athenes M, Bellon P, Martin G and Haider F 1996 Acta Mater. 444739

[26] Khachaturyan A G 1983 Theory of Structural Phase Transformations in Solids (New York: Wiley)

[27] Finel A, Mazauric V and Ducastelle F 1990 Phys. Rev. Lett. 651016

[28] Pearson W B 1972 The Crystal Chemistry and Physics of Metals and Alloys (New York: Wiley)

[29] Karlik M 1997 Mater. Sci. Eng. A 234-236 212

[30] Hasaka M 1980 Trans. JIM 21660

[31] Inden G 1974 Acta Metall. 22945

[32] Raviprasad K and Chattopadhyay K 1993 Acta Metall. Mater. 41609

[33] Bell J M 1987 Physica A 14222

[34] Polatoglou H M 1996 Mater. Sci. Eng. B 37177

[35] Beiden S V and Vaks V G 1992 Phys. Lett. A 163209

[36] Wang Y, Chen L-Q and Khachaturyan A G 1992 Phys. Rev. B 4611194

[37] Beiden S V, Samolyuk G D, Vaks V G and Zein N E 1994 J. Phys.: Condens. Matter 68487

[38] Koyama T, Miyazaki T and Mebed A E M 1995 Metall. Mater. Trans. A 262617

[39] Pollock T M and Argon A S 1994 Acta Metall. Mater. 421859 